\title{
Die würm- und postwürmglazialen Terrassen des Lech und ihre Bodenbildungen
}

\author{
Von Theodor Diez, München
}

Mit 6 Abbildungen und 6 Tabellen im Text

\begin{abstract}
$\mathrm{Z}$ us a mme n fas u n g. Die Terrassenlandschaft des Lech zwischen Landsberg und Schongau umfaßt wenigstens 16 scharf ausgeprägte würm- und postwürmglaziale Terrassentsufen. Die obersten (ältesten) sechs Stufen sind mit den Endmoränenständen des Lechgletschers zu parallelisieren, die zweitjüngste Stufe ist durch begrabene frührömische Funde relativ exakt datierbar. Die Grenze zwischen Spät- und Postglazial wird mit Hilfe bodenkundlicher Vergleichsuntersuchungen ermittelt.

Die ungestörten Maximalbodenbildungen der einzelnen zeitlich fixierten Terrassenstufen werden beschrieben. Erhebliche Entwicklungsunterschiede zwischen den Böden der Niederterrassen und denen der Postglazialterrassen sind nur durch eine bereits im Spätglazial erfolgte, starke Bodenbildung erklärbar. Ein Vergleich von Acker- und Waldprofilen zeigt die starke Veränderung der Böden unter dem Einfluß der landwirtschaftlichen Bodennutzung.

$\mathrm{Summary}$. The terrace-landscape of the river Lech between Landsberg and Schongau includes at least 16 sharply developed terraces of würm- and postwürm-glacial age. The top (oldest) 6 terrace levels can be connected with the terminal moraine stages of the Lechglacier; the youngest but one level is exactly to be dated by burried early roman findings. The limit between late- and postglacial times is found through comparative investigations on soils.

The undisturbed maximum soil profiles of the individual, temporally fixed terrace levels are described. Considerable differences in development between soils of the high glacial and those of the postglacial terraces are only explainable by a strong soil development taking place already in late glacial times. Comparing cropland and forest soil profiles the intense change of soils under the influence of agricultural use is becoming obvious.
\end{abstract}

\section{Einleitung}

Die Bodentypen im Sinn der genetischen Bodenlehre sind das Ergebnis des Zusammenwirkens mannigfacher Faktoren, von denen das Ausgangsmaterial und die Zeitdauer der Bodenbildung unter bestimmten Klimaverhältnissen die wichtigsten sind. Während das Ausgangsmaterial in den meisten Fällen leicht zu bestimmen ist, gibt es über die Zeitdauer meist nur vage Anhaltspunkte.

Exakte Vorstellungen über die Bildungsgeschwindigkeit unserer Böden sind nur auf stabilen, d.h. von Erosion und Akkumulation freien Landoberflächen bekannten Alters zu gewinnen. Unter diesen Gesichtspunkten bietet die Terrassenlandschaft des Lech zwischen Schongau und Landsberg ein geradezu ideales Studienobjekt: Die jungpleistozänpostglaziale Talbildung hat hier eine Terrassentreppe von wenigstens 16 scharf gegeneinander abgesetzten Terrassenstufen entstehen lassen, von denen die jüngeren jeweils in die älteren „eingeschachtelt" sind. Die Verknüpfung der älteren Terrassen mit den Endmoränenwällen einerseits und eine durch Artefakte genau datierbare mittelrömische Terrasse bei Epfach andererseits, stellen relativ exakte Zeitmarken für die zeitliche Fixierung der Terrassen und ihrer Bodenbildungen dar.

Die Mechanik der Talbildung der Alpenflüsse im endmoränennahen Bereich ist von C. Troll (1926) mit all ihren Eigentümlichkeiten umfassend beschrieben worden. Wenn trotzdem der Terrassenbildung am Lech in dieser Arbeit ein eigenes Kapitel gewidmet wird, so deshalb, weil für die Beurteilung des Alters der Böden eine Terrassenfeingliederung und ihre Parallelisierung mit dem würm-postglazialen Klimaablauf die Voraussetzung bildet. 


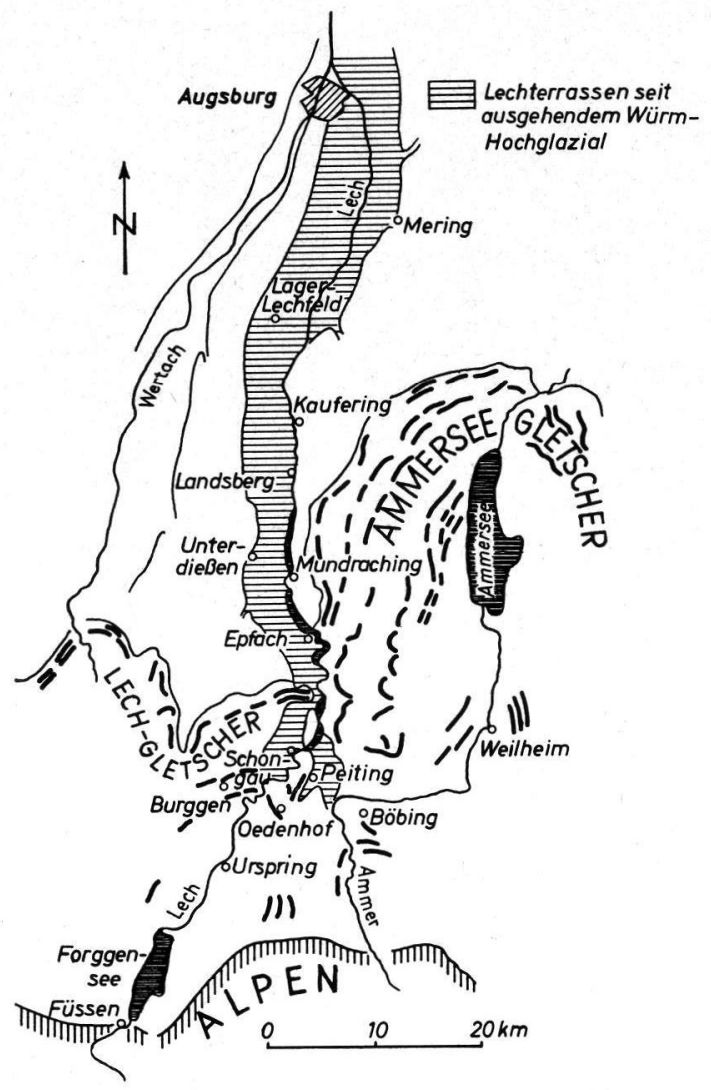

Abb. 1. Das Lechtal im Alpenvorland in Beziehung zur würmzeitlichen Vergletscherung.

Den Anstoß zu dieser Arbeit gab die vom Verfasser in den Jahren 1964/65 durchgeführte Kartierung der Lechterrassen auf Blatt 7931 Landsberg a. L. im Rahmen der bodenkundlich-geologischen Landesaufnahme 1:25000 von Bayern. Um die Terrassen und ihre Bodenbildungen zeitlich einordnen zu können, war es notwendig, die Untersuchungen nach Süden auszudehnen, d. h. eine Verknüpfung der Terrassen bei Landsberg mit den Endmoränen bzw. ihre Parallelisierung mit der mittelrömischen Terrasse bei Epfach herzustellen.

Die Ergebnisse werden nachstehend in drei Teilen abgehandelt. Der erste Teil behandelt die Flußgeschichte des Lech seit dem Maximalstand der würmzeitlichen Vergletscherung, der zweite Teil befaßt sich mit den Bodenbildungen der einzelnen Terrassenstufen in chronologischer Reihenfolge. Im dritten Teil wird versucht, die geomorphologisch-bodenkundlichen Untersuchungsergebnisse im Hinblick auf die Tal- und Bodenentwicklung, getrennt nach spät- und postglazialer Entwicklung, zu interpretieren.

\section{A. Die Parallelisierung der würm- und postwürmglazialen Lechterrassen zwischen Schongau und Lager Lechfeld}

Etwa $5 \mathrm{~km}$ nördlich von Schongau durchbricht der Lech die äußerste Endmoräne des Lechgletschers. Die Höhendifferenz zwischen seiner ältesten, mit der Würm-Endmoräne verknüpften Terrasse und seinem heutigen Hochwasserbett beträgt an dieser Stelle rund $70 \mathrm{~m}$. $20 \mathrm{~km}$ weiter nördlich bei Landsberg-Kaufering hat dieser Höhenunterschied be- 
reits auf etwa $24 \mathrm{~m}$ abgenommen (Abb. 2). Die zwischen Schongau und Landsberg durch klare Terrassenkanten und große Sprunghöhen äußerst scharf ausgeprägten Terrassenstufen verschwinden nördlich Landsberg zum größten Teil. Die Sprunghöhen nehmen ab, die Terrassenkanten werden unscharf, die älteren Stufen verlieren sich im Niveau der jüngeren. $16 \mathrm{~km}$ nördlich Landsberg, auf der Höhe von Lager Lechfeld, sind nur noch drei eindeutig erkennbare Terrassenstufen vorhanden, die, wenn man sie nach den Sedimentdeckschichten der Oberflächen einstuft, bereits alle ins Postglazial gestellt werden müssen.

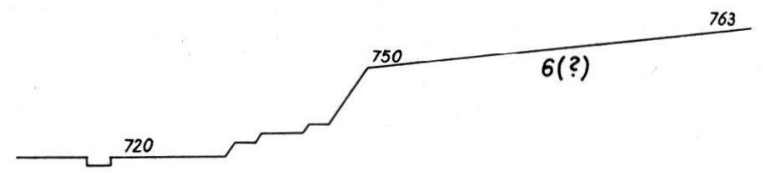

Urspring
(Steingaden)
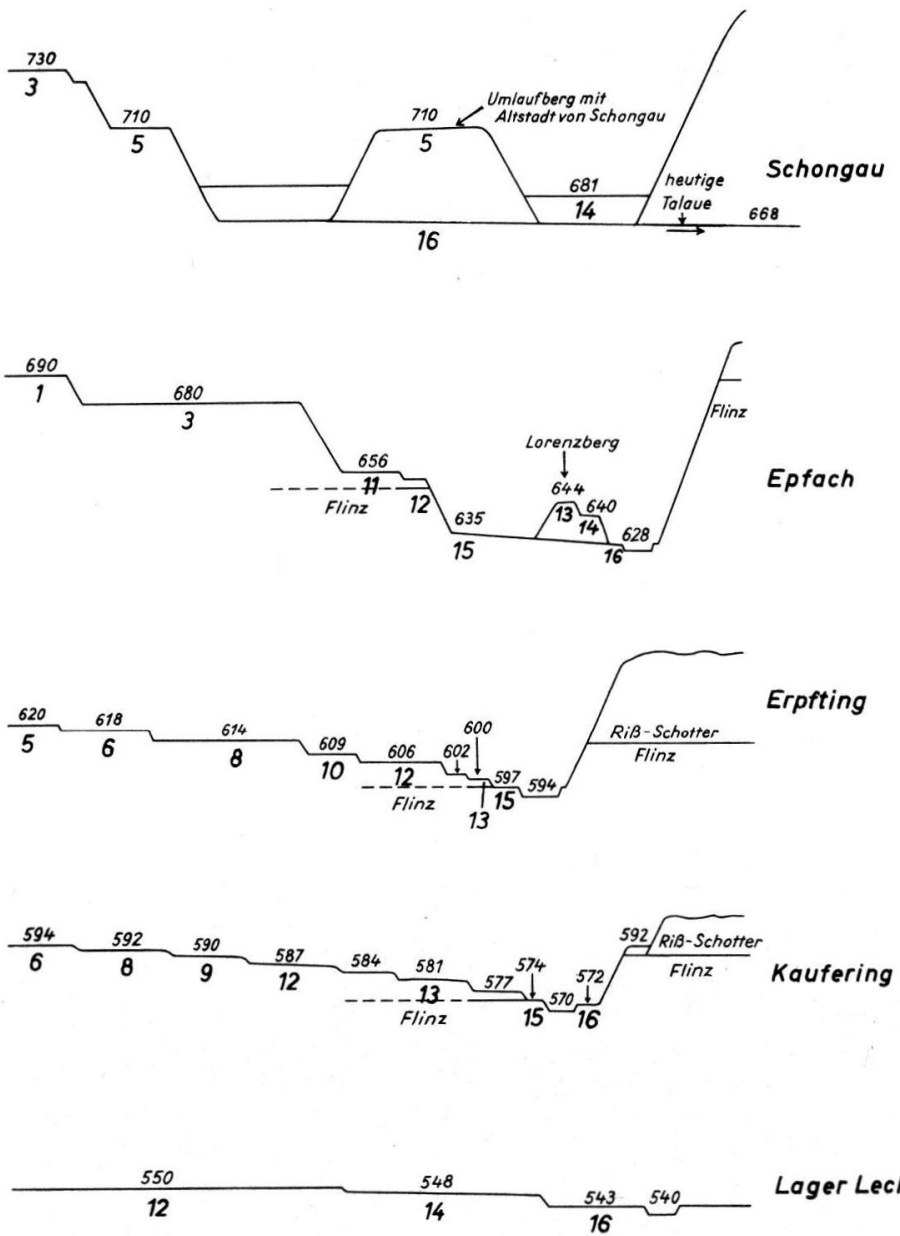

Lager Lechfeld

Abb. 2. Die würm- und postwürmglaziale Terrassenabfolge des Lechtales, dargestellt in Querprofilen verschiedener Talabschnitte zwischen Urspring (Steingaden) und Lager Lechfeld (die über dem Terrassenprofil angegebenen Zahlen kennzeichnen die Höhen über NN, die darunterstehenden die Terrassenstufen wie in Abb. 3). 


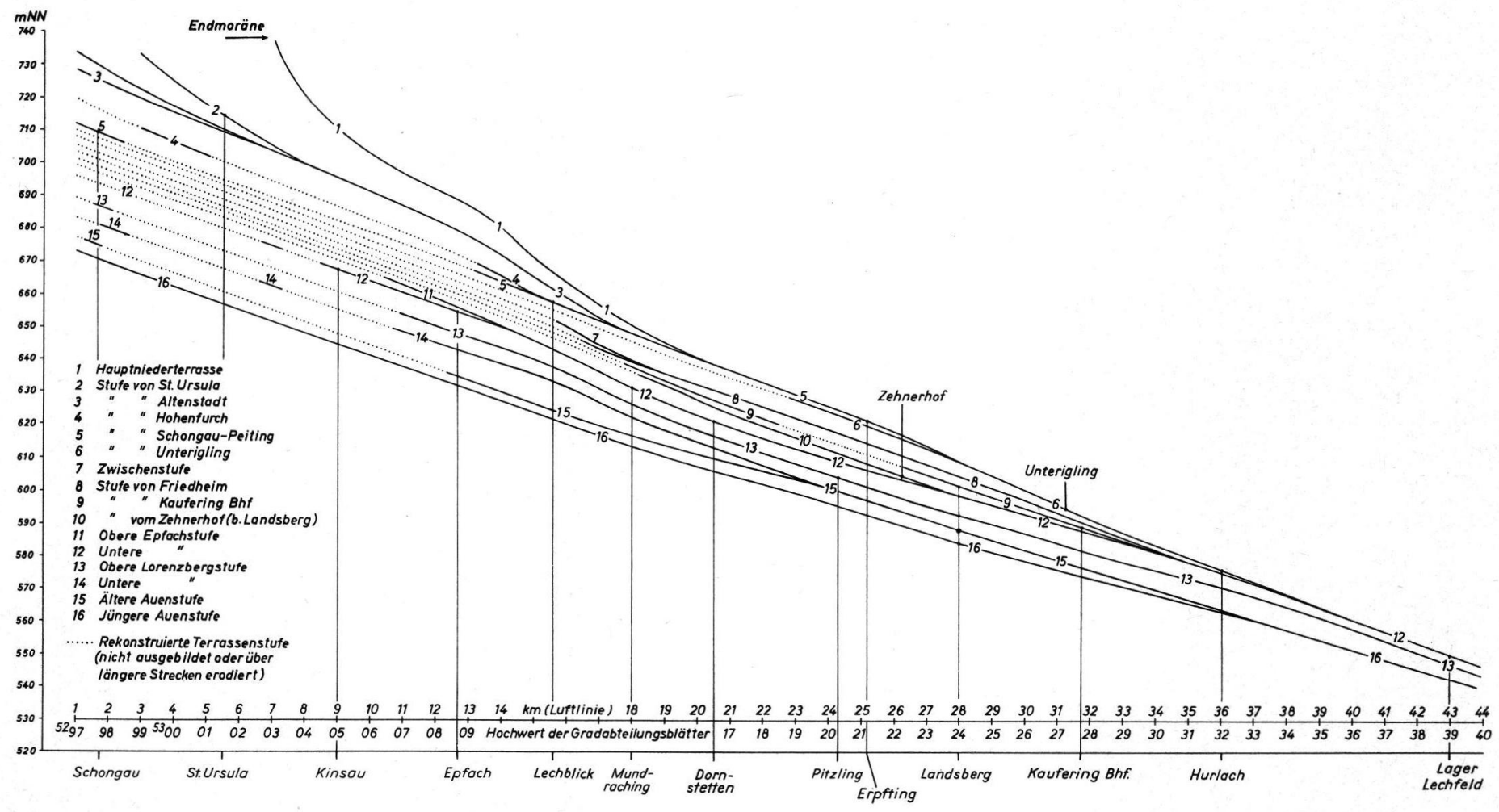

Abb. 3. Die würm- und postwürmglaziale Terrassenabfolge des Lechtales zwischen Schongau und Lager Lechfeld (Längsprofil). 
Die südwestlich Schongau stark einsetzende Eintiefung des Lech, welche die Voraussetzung für die reiche Terrassengliederung bildet, ist auf die Überhöhung des Geländes durch die Endmoränenablagerungen des Lechgletschers zurückzuführen sowie auf das Bestreben des Flusses, sein Gefälle wieder auszugleichen.

Die Ursache der Terrassenbildung wird in einem klimatisch bedingten Wechsel zwischen Tiefen- und Breitenerosion gesehen. Für die ältesten Terrassen, die sich mit Endmoränen verknüpfen lassen, ist die Terrassenentstehung relativ klar: Die Tieferlegung des Flußbettes in die von den Schmelzwässern zuvor aufgeschütteten Sedimente erfolgte während des Rückwanderns der Gletscherstirn. Seitenerosion, die zum Schluß in schwache Aufschotterung überging (SCHÄFER 1950), herrschte zu Zeiten des Verweilens des Eisrandes. Der Entstehung eines Rückzugsmoränenkranzes entspricht somit zeitlich die Ausbildung eines Talbodens, der nach neuerlichem Erosionseinschnitt zur Terrasse wurde.

Die Rekonstruktion des Terrassenverlaufs stößt insofern auf Schwierigkeiten, als infolge des Mäandrierens des Flusses einzelne Terrassen häufig auf große Strecken erodiert oder bei kerbtalartigem Einschneiden in bestimmten Talabschnitten überhaupt nicht zur Ausbildung gelangt sind. Kompliziert wird die Terrassengliederung noch durch die im Endmoränenvorland häufige Terrassenkreuzung.

In Abb. 3 ist der Verlauf der einzelnen, seit dem Würm-Hochglazial gebildeten Terrassenstufen des Lech zwischen Schongau und Lager Lechfeld im Längsprofil dargestellt. Die Parallelisierung nichtzusammenhängender Terrassenabschnitte erfolgte auf Grund von Höhenvergleichen und durch Vergleich der Bodenbildungen. Der Bereich der Terrassenkreuzung wurde durch aufmerksames Verfolgen der Terrassenkanten und Gefällsverhältnisse ermittelt.

\section{Ausbildung und Erstreckung der einzelnen Terrassenstufen}

a) Hautniederterrasse (1)

Sie geht unmittelbar aus dem Übergangskegel der äußersten Endmoräne des Lechgletschers hervor und erstreckt sich in einer Breite von 1,5-3 km bis etwas Unterdießen, wo sie von den Sedimenten jüngerer Terrassen überlagert wird. Ihr Gefälle von anfangs über 10\% nimmt gegen Unterdießen auf etwa $5 \%$ ab.

b) Die Stufen von St. Ursula (2) und Altenstadt (Römerau) (3)

Die Stufe von Altenstadt läßt sich mit dem ersten stark ausgeprägten Rückzugsstadium des Lechgletschers, d.h. dem Moränenkranz nördlich Burggen verknüpfen. Westlich Schongau noch ein mächtiges Schotterfeld bildend, sammeln sich die Schmelzwässer zwischen Altenstadt und Hohenfurch, um zwischen Hohenfurch und Kinsau den äußersten Endmoränenwall zu durchbrechen. Bei Kinsau hat sich die Stufe von Altenstadt bereits $15 \mathrm{~m}$ tief in die Hauptniederterrasse eingeschnitten. Auf der Höhe von Asch verliert sie sich in der jüngeren Terrassentsufe von Schongau-Peiting. Ihr durchschnittliches Gefälle beträgt $5 \%$.

Zwischen Schongau und Hohenfurch verläuft die B 17 zum größten Teil auf einem alten Talboden, der auf Grund seiner Höhenlage einer Eisrandlage zwischen den beiden ausgeprägten äußeren Endmoränenwällen angehören muß. Darauf hat bereits C. RATHJENS (1951) hingewiesen. Diese, nach der auf ihr stehenden Kapelle St. Ursula benannten Terrasse, ist nur von sehr kurzer Erstreckung. Unmittelbar nach dem Durchbruch durch den Endmoränwall verschwindet sie unter der viel ausgeprägteren Terrasse von Altenstadt.

c) Stufe von Hohenfurch (4)

Nördlich des Umlaufberges von Schongau (etwa dort, wo heute das neue Kreiskrankenhaus steht), beginnt ein rund $12 \mathrm{~m}$ in die Stufe von Altenstadt eingeschnittenes, stark 
mäandrierendes Trockental, das der Lech unmittelbar vor seiner Ablenkung zu seinem heutigen Durchbruchstal benutzte. Nach dem auf seiner Talsohle stehenden Dorf Hohenfurch wurde diese Stufe von C. Troll (1954) „Stufe von Hohenfurch" benannt. Ihre Bildung erfolgte während des Eisrückzuges von dem Burggener Moränenzug (1. Rückzugsstadium) auf den Endmoränenwall von Litzau-Oedenhof (2. Rückzugsstadium). Die Terrassenstufe ist zwischen Schongau und dem äußersten Endmoränzug als sehr markantes Trockental ausgeprägt, dann fehlt von ihr über eine Entfernung von $\mathrm{rd} .7 \mathrm{~km}$ jede Spur. Erst nördlich von Epfach, kurz vor ihrer Überlagerung durch die Stufe von Schongau-Peiting, ist noch ein schmaler Terrassenrest erhalten, der dieser Terrassenstufe angehören dürfte.

d) Stufe von Schongau-Peiting (5)

Während der Bildung der nächsten, in ihrem Niveau nur wenig tiefer liegenden Terrassenstufe von Schongau-Peiting hatte der Lech bereits sein Hohenfurcher Tal verlassen und benutzte die Enge von Finsterau. C. Troll (1954) erklärt diese plötzliche Flußlaufverlegung wie folgt:

„... beim weiteren Rückzug auf die nächst jüngeren Eisrandlagen teilten sich Lech- und Ammerseegletscher vollständig und zwischen ihnen kam viel weiter südlich in der Gegend von Bayersoien der kleine Ammergletscher zur freien Entfaltung. Die Eisränder lagen dann am Lechgletscher bei Butzau, am Ammerseegletscher bei Böbing. In dem großen, eisfrei gewordenen Raum sammelten sich Schmelzwasser der drei Gletscher in einem Netz von Urstromtälern: das Ammertal von Rottenbuch-Peiting, das alte Illachtal von Kirchberg nach Peiting und das Kellerhofer Tal. Sie liefen alle bei Peiting zusammen und führten von dort über dem heutigen Lechtal gegen Kinsau. Diese gesammelten Schmelzwasser hatten die Kraft, bei Peiting durch Tieferschalten in die Hohenfurcher Terrasse eine breite Schotterrinne auszufurchen."

Die Terrassenstufe von Schongau-Peiting wurde also gebildet, als der Lechgletscher schon etwa die Hälfte des hochglazial eisbedeckten Vorlandes wieder freigegeben hatte. Die von C. Troll vorgenommene Parallelisierung des Schongau-Peitinger Schotterniveaus mit dem von Kinsau-Epfach und die damit angenommene, starke Einschneidung des Flusses kann allerdings nicht übernommen werden. Die Kinsauer Terrasse (= Untere Epfachstufe) ist sehr viel jünger. Das geht nicht nur aus den Gefällekurven (Abb. 3), sondern auch aus den Bodenbildungen hervor. Dem Schongau-Peitinger Niveau ist vielmehr die viel ausgeprägtere Terrasse gleichzustellen, die sich zwischen dem Café Lechblick und Mundraching zunächst mit der Stufe von Altenstadt und später, bei Unterdießen, mit der Hauptniederterrasse kreuzt. Im Talabschnitt Schongau-Epfach wurde die Terrasse infolge der starken Mäandrierung des späteren Flußlaufes vollständig erodiert. Erst nördlich Epfach setzt sie kurz hinter dem Wiedererscheinen der Stufe von Hohenfurch als zunächst schmale Terrassenstufe wieder ein. Im Talabschnitt Pitzling-Landsberg bedecken ihre Sedimente ein über $5 \mathrm{~km}$ breites Tal mit einem durchschnittlichen Gefälle von ca. 4\%

e) Stufe von Unterigling (6)

Zwischen Unterdießen und Ellighofen zeigt eine neu auftretende, etwa $2 \mathrm{~m}$ tief in die Schongau-Peitinger Terrasse eingeschnittene Stufe die nächste Phase der Tieferlegung des Flusses an. Nach dem auf ihr liegenden Ort Unterigling soll sie als „Stufe von Unterigling " bezeichnet werden. Sie kreuzt die Schongau-Peitinger Terrasse in Höhe des Zehnerhofes am südlichen Stadtrand von Landsberg. Mit einer Talbreite von über $5 \mathrm{~km}$ erstreckt sie sich etwa bis Hurlach, wo sie von jüngeren Ablagerungen überdeckt wird. Ihr durchschnittliches Gefälle beträgt 4,1\% westl. und nordwestl. Landsberg ergaben Gesamtschottermächtigkeiten etwa zwischen 15 und 30 Metern. Über die Aufschotterungsmächtigkeiten der einzelnen Stufen können keine Angaben gemacht werden.

Die Verknüpfung dieser Terrasse mit dem zugehörigen Endmoränenzug ist nur indirekt möglich. Nach C. Troll (1925) folgt auf den 3. Rückzugshalt, dem die Schongau- 
Peitinger Stufe entspricht, als nächstjüngere Gletscherstillstandsphase das sog. AmmerseeStadium. Die dem Ammersee-Stadium zugeordneten Endmoränenwälle liegen für den Ammerseegletscher bei Weilheim, für den Lechgletscher am Nordrand des Füssener Bekkens. Die Bodenbildungen der Füssener Moränen (DIEz 1964, S. 133) entsprechen unter Berücksichtigung des Niederschlagsgefälles ungefähr denen der Stufe 6, so daß sowohl von der Geomorphologie als auch von der Bodenkunde her eine zeitliche Gleichstellung der Unteriglinger Terrasse mit dem Ammersee-Stadium gerechtfertigt ist.

f) Stufe von Friedheim (8)

Unmittelbar nach dem Prallhang beim „Lechblick“ beginnen unterhalb der SchongauPeitinger Stufe zwei weitere ausgeprägte Terrassenstufen. Die obere (7) besitzt sehr starkes Gefälle (ca. 7\%o0) und verschwindet bereits nach $1 \mathrm{~km}$ unter der unteren Stufe (8), die sich fast lückenlos etwa vom Lechblick bis Kaufering erstreckt. Vom Lechblick bis Landsberg verläuft die B 17 auf ihrem Niveau. Ihr Gefälle beträgt etwa 3,6\% \% .

Die älteren Terrassen bis einschließlich der Stufe von Friedheim sind alle sehr klar gegeneinander abgesetzt und ohne Schwierigkeiten über längere Strecken zu verfolgen. Demgegenüber ist die Abgrenzung und Paralleliserung der jüngeren Terrassenabschnitte infolge der starken Zerstückelung, der geringeren Höhendifferenzen und dem unausgeglicheneren Relief wesentlich schwieriger. Zahlreiche, morphologisch gut ausgebildete Terrassenkanten erstrecken sich häufig nur über wenige 100 Meter, um ebenso allmählich, wie sie begonnen haben, wieder im Hauptniveau der Terrassen auszuklingen. Für die nachfolgende Gliederung der Lechterrassen bleiben sie unberücksichtigt.

g) Die Stufen von Kaufering Bhf (9) und vom Zehnerhof (10)

Die Terrassenstufe von Kaufering Bhf erscheint erstmals auf der Höhe von Mundraching (die höchstgelegenen Häuser der Ortschaft Seestall liegen noch auf ihr). Sie gliedert sich rasch in eine Reihe kleinerer Terrassenstufen mit Sprunghöhen von weniger als 2 Meter, die meist nach kurzem Verlauf wieder verschwinden. Weiter nördlich liegen die westliche Neustadt von Landsberg sowie Kaufering Bhf auf ihrem Niveau. Ihr durchschnittliches Gefälle beträgt 3,1\%

Aus dem Terrassenkomplex macht sich etwa $1 \mathrm{~km}$ nördlich Seestall eine neue Terrassenstufe selbständig, die sich bis zur Stadtgrenze von Landsberg erstreckt, wo sie sich mit der nächst jüngeren Terrasse kreuzt. Der Zehnerhof südlich Landsberg, nach dem sie benannt sein soll, liegt auf ihrem Niveau.

Die beiden, auf Grund ihrer geringen Höhendifferenz und ihrer Bodenbildungen eng zusammengehörigen Terrassenstufen zeigen erstmalig eine mehr oder weniger zusammenhängende schluffige bis schluffig-feinsandige Deckschicht (im folgenden kurz „Flußmergel“ genannt) von etwa $1-2 \mathrm{dm}$ Mächtigkeit über dem Schotterkörper. Diese für alle jüngeren Terrassen kennzeichnende Flußmergeldecke dürfte einerseits auf das geringer gewordene Terrassengefälle (geringere Strömungsgeschwindigkeit $\rightarrow$ Sedimentation feinerer Korngrößen), andererseits auf die zunehmende Unterschneidung der Flinzgrenze und den damit verbundenen verstärkten Anfall feinsandig-schluffigen Materials zurückzuführen sein.

h) Obere und Untere Epfachstufe $(11,12)$

Der Ort Epfach liegt auf zwei nur wenige Meter gegeneinander abgesetzten Terrassenstufen, die von K. BrunNacker (1964) als Obere und Untere Epfachstufen bezeichnet wurden. Die Obere Epfachstufe läßt sich von Apfeldorf bis etwa $1 \mathrm{~km}$ nordwestlich Epfach verfolgen, wo sie unter der Unteren Epfachstufe verschwindet. Die Untere Epfachstufe erscheint nach dem Durchbruch des Lech durch die Endmoräne erstmalig bei Kinsau als breite Terrasse. 
K. Brunnacker (1959, S. 80 und 1964, S. 142) hat die Epfachstufen unter Berufung auf C. Troll zu den "jüngeren“, aber immer noch unter eiszeitlichen Bedingungen entstandenen Terrassen gestellt. Als Beweis dafür führt er zwei auf das Niveau der Unteren Epfachstufe ausmündende asymmetrische Seitentälchen an. In Frage kommen dafür nur die beiden Seitentälchen links des Lech im Talabschnitt Epfach-Lechblick. Eine genauere Untersuchung dieser Tälchen zeigte jedoch, daß sie ursprünglich hoch über dem Niveau der Epfachstufe ausstrichen. In den nach Art glazialer Hängetäler ausmündenden, breiten Talboden hat die spätere Erosion eine schmale Rinne eingekerbt, über die der Anschluß der asymmetrischen, oberen Talstrecke zum Niveau der Epfachstufe hergestellt wird. Daraus ergibt sich, daß der übersteilte, kerbtalartige Mündungsabschnitt jünger ist als der breitere und flacher geneigte Oberlauf.

Die asymmetrischen Tälchen führen zwar normalerweise kein Oberflächenwasser, doch ist es durchaus nicht abwegig, anzunehmen, daß wolkenbruchartige Regenfälle in postglazialer Zeit die oben beschriebene Erosionswirkung hervorrufen konnten. Wahrscheinlich wurde das Mündungsgebiet der asymmetrischen Tälchen auch durch Menschenhand verändert, da sich die Tälchen für die Anlage von Wegen zur Überwindung des steilen Terrassenabfalls geradezu anboten.

Die asymmetrischen Tälchen bei Epfach sind somit nicht als Beweis für das eiszeitliche Alter der Epfachstufen zu werten. Als Bildungen des ausgehenden Spätglazials mit ihrem hängetalartigen Ausstreichen hoch über dem Niveau der nächstfolgenden Terrasse weisen sie im Gegenteil darauf hin, daß die Untere Epfachstufe bereits weit ins Postglazial zu stellen ist.

Die Verfolgung der Terrasse nördlich des Café Lechblick wird durch die starke Mäandrierung des Flusses und die dadurch bedingte Erosion langer Talabschnitte sehr erschwert. Nach einer kurzen Talstrecke mit relativ starkem Gefälle und starkem Einschneiden (etwa zwischen den Staustufen 11 und 12) erreicht die Terrasse das Gefälle des heutigen Talbodens (ca. 3\%or) und überlagert talabwärts die Stufen vom Zehnerhof in der Höhe von Landsberg, die von Kaufering Bhf und Friedheim wenige Kilometer nördlich Kaufering. Bei Kloster Lechfeld schließlich wird sie mit der Stufe von Igling niveaugleich, die sie jedoch nur noch mit einer geringmächtigen Flußmergelschicht überdecken konnte( im allgemeinen weniger als $2 \mathrm{dm}$ ).

Im Talabschnitt Epfach-Dornstetten beträgt die Mächtigkeit des Schotterkörpers über dem Flinzuntergrund im Mittel etwa $1-3 \mathrm{~m}$; sie steigt talabwärts an. Eine geringmächtige Flußmergeldeckschicht von $1-2 \mathrm{dm}$ ist für die gesamte Terrassenstufe die Regel.

i) Obere und Untere Lorenzbergstufe $(13,14)$

Die nach dem Lorenzberg bei Epfach von K. BRUnNACKer (1964) so benannten, nächstjüngeren Terrassenstufen sind wiederum nur sehr lückenhaft erhalten. Die Obere Lorenzbergstufe, auf der die Kapelle steht, läßt sich mit einem Terrassenrest westlich Schongau (oberhalb des Lexenbaches) parallelisieren, die Untere Lorenzbergstufe, auf der das römische Kastell stand, ist gleich alt mit dem Talboden der Lechschleife bei Schongau, durch die der Umlaufberg geschaffen wurde, auf dem heute die Schongauer Altstadt steht.

Die Obere Lorenzbergstufe erstreckt sich bei einem der heutigen Talaue angenäherten Gefälle bis über Lager Lechfeld hinaus, ohne eine der älteren Terrassen zu kreuzen. Die Untere Lorenzbergstufe verliert sich etwa in der Höhe von Pitzling unter den Sedimenten der Älteren Auenstufe.

Die Gesamtmächtigkeit des Terrassenkörpers über dem Flinzuntergrund dürfte, wie bei der Unteren Epfach-Terrasse, im Durchschnitt zwischen 1 und $3 \mathrm{~m}$ liegen. Die Flußmergeldeckschicht über dem Schotterkörper ist gewöhnlich etwas mächtiger entwickelt als auf den älteren Terrassen.

k) A 1 tere und Jüngere Auenstufe $(15,16)$

Die schon bei der Oberen Lorenzbergstufe beobachtete, über den gesamten Untersuchungsabschnitt etwa gleichbleibende Tiefenerosion setzt sich im Lauf der weiteren Talbildung fort. Nach ihren Bodenbildungen lassen sich neben einer älteren Auenstufe eine ganze Reihe jüngerer Auenstufen ausscheiden, die jedoch infolge der häufigen Überstauung und mangels signifikanter Bodenunterschiede über längere Strecken nicht mehr zu parallelisieren sind. 
Die Altere Auenstufe ist für die zeitliche Einordnung der Terrassen insofern von Bedeutung, als ihr Alter durch historische Funde am Fuß des Lorenzberges gut datierbar ist. Der Lorenzberg, schon in frührömischer Zeit ein strategisch wichtiger Militärstützpunkt zur Sicherung des Lechüberganges bei Epfach (dem römischen Abodiakum), geht im Süden mit scharfem Geländeknick in die Ältere Auenstufe über. Eine Querschnittsgrabung seitens des Instituts für Vor- und Frühgeschichte, München, erbrachte folgende Ergebnisse: „Unmittelbar am Fuße des Berges fanden sich in einer Tiefe von 2,75 m unter dem heutigen Niveau große Rollsteine in unregelmäßiger Lagerung auf dem gewachsenen Flinz. Auf, zwischen und unter den Rollsteinen lagen verschiedene frührömische Kleinfunde verstreut. Darüber lagerte ein starkes fluviatiles Sediment in Form von nach oben immer feiner werdendem Kies und feinstem Flußsand. Die Ablagerung erreichte eine Stärke von fast $2,50 \mathrm{~m}$. In diese wurden in spätrömischer Zeit zwei Spitzgräben eingetieft “ (UlbERT 1965, Abb. 4).

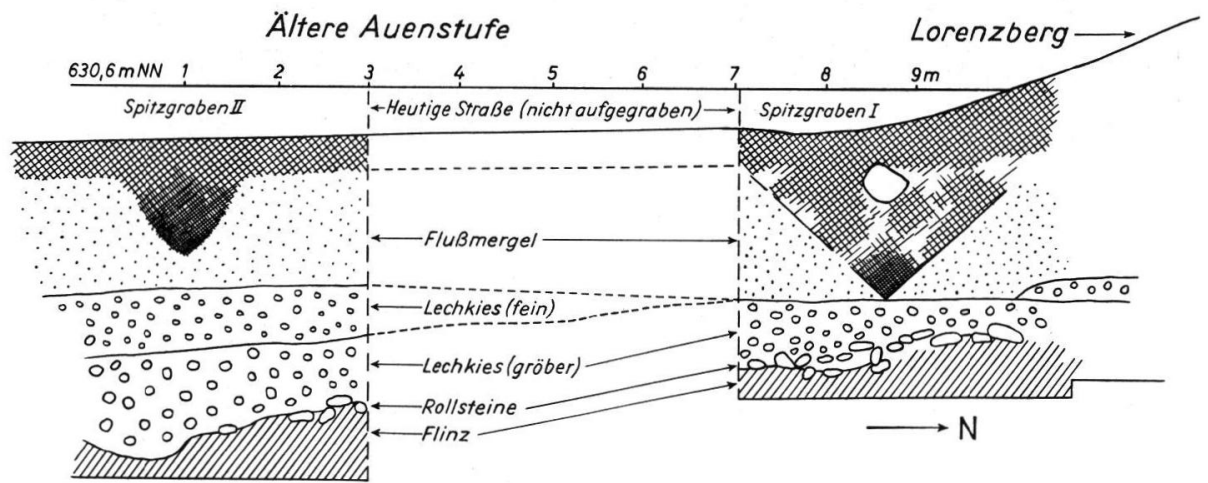

Abb. 4. Sedimentfolge der Alteren Auenstufe und Relikte römischer Befestigungsanlagen am Südhangfuß des Lorenzberges bei Epfach (maßstabgetreues Profil aus den Grabungsunterlagen des Instituts für Vor- und Frühgeschichte der Universität München. Westprofil, Schnitt I).

Der Grabungsbefund zeigt deutlich, daß der Lech in frührömischer Zeit am Südhang des Lorenzberges entlangfloß und in den folgenden Jahrhunderten die Ältere Auenstufe aufschüttete. Spätestens im 4. nachchristlichen Jahrhundert mußte er seinen Lauf unter gleichzeitiger Tieferlegung weiter nach Osten, d.h. in die Nähe seines heutigen Bettes verlagert haben, wie die Anlage der Spitzgräben (Befestigungsanlagen) beweist. Die erheblich kleineren Ausmaße des Spitzgrabens II (Abb. 4) lassen es denkbar erscheinen, daß durch spätere Hochfluten ein Teil der Terrassensedimente wieder abgetragen wurde. Jedenfalls darf bei der geringen Höhendifferenz zwischen heutigem Flußwasserspiegel und Alterer Auenstufe (sie beträgt im Mittel nicht mehr als 3-5 m) angenommen werden, daß starke Hochwässer das Gelände gelegentlich auch noch bis in jüngere Zeit überschwemmt haben.

\section{Zusammenfassende Darstellung der Talentwicklung seit dem ausgehenden Würm-Hochglazial}

Die in dieser Arbeit vorgenommene Rekonstruktion der Talentwicklung des mittleren Lech auf Grund der Terrassenparallelisierung fügt sich im grundsätzlichen gut in die schon lange feststehenden Erkenntnisse der jungpleistozänen Talbildung im Endmoränen- und Endmoränenvorlandbereich (C. Troll 1926, I. Schäfer 1950, H. Graul 1962 u. a.):

1. Auf eine Periode der Tiefen- und Breitenerosion im Riß/Würm-Interglazial und während des würmglazialen Eisvorstoßes folgte eine Periode der Aufschotterung, die ihren Höhepunkt erreicht hatte, als der Lechgletscher begann, sich von seiner maximalen Eis- 
randlage zurückzuziehen. Die von den Endmoränenwällen kommenden Schmelzwässer schufen die steil nach Norden einfallende Hauptniederterrasse (1) mit einem ersten Schwemmkegel in Höhe Epfach-Lechblick.

2. Dem schrittweisen Gletscherrückzug zum Alpenrand entsprechen die Terrassenstufen 2-6. Sie zeigen, gemessen am Betrag der Tiefenerosion, eine rasch abnehmende Erosionsleistung parallel zum Eisrückzug, die einerseits auf die Gefälleverflachung, andererseits auf die immer noch beträchtliche Schuttlieferung der Gletscher zurückzuführen ist.

3. Einem jeder Terrassenstufe zugehörigen Talabschnitt der Einschneidung entspricht talabwärts ein solcher der Aufschotterung. Die im Einschneidungsabschnitt ausgeräumten Sedimente überlagern die Sedimente einer älteren Stufe unter gleichzeitiger Talverbreiterung in dem Augenblick, in dem das Niveau der jüngeren Stufe das der älteren erreicht. Die so entstandenen Talformen sind im Sinn von C. Troll (1926) klassische Trompetentäler.

Einschneidung und Aufschotterung sind im Spät- und Postglazial nicht nur räumlich, sondern auch zeitlich getrennte Vorgänge. Bis in jüngste Zeit wird die Bildung einer Terrassenstufe durch eine Periode der (wenn auch meist nur geringmächtigen) Sedimentation abgeschlossen, auch wo die Terrassen nach ihrer Morphologie reine Erosionsterrassen zu sein scheinen. Dies geht aus der allgemeinen Verbreitung der Flußmergeldeckschichten auf den jüngeren Terrassenstufen hervor.

4. Eigenartig ist die starke Gefällestrecke zwischen dem Café Lechblick und Mundraching, die von der Hauptniederterrasse bis zur Oberen Lorenzbergstufe allen Terrassen gemeinsam ist. Diese Erscheinung hängt möglicherweise mit einer schon vor der würmglazialen Talverschüttung vorhanden gewesenen, alten Talfurche zwischen Epfach und Lechblick zusammen (J. KNAUER 1929, S. 5), deren Füllmassen der Tiefenerosion einen stärkeren Widerstand entgegensetzten (Nagelfluh?) als der oberhalb und unterhalb hoch anstehende, weiche Tertiäruntergrund.

5. Die Überschneidung älterer Terrassenniveaus mit jüngeren hat zur Folge, daß die Oberfläche älterer Terrassenstufen talabwärts unter den Sedimenten jüngerer Stufen begraben wird. Die junge Sedimentation erreicht unmittelbar nach der Terrassenkreuzung ihre größte Mächtigkeit (Schwemmkegelbildung) und klingt talabwärts allmählich ab. Eine Abgrenzung der älteren Sedimente von den jüngeren ist nur dort möglich, wo der Sedimentcharakter wechselt (z. B. Flußmergel über Schotter) bzw. wo unter einer jungen Sedimentdecke Bodenrelikte der älteren Terrassenstufe erhalten sind (s. S. 120).

6. Der Verlauf der Gefällekurven in Abb. 3 zeigt anschaulich die Problematik der Alterseinstufung einzelner, aus dem Zusammenhang gelöster Terrassenabschnitte. Am deutlichsten wird dies für die sog. Niederterrasse. Die auf den vorhandenen Kartenwerken (Schäfer, I.: Geologische Karte von Augsburg und Umgebung 1 : 50 000, München 1956; Geologische Karte von Bayern 1:500 000, II. Aufl., München 1964) als Niederterrasse ausgeschiedene Fläche umfaßt eine Reihe von Oberflächen, deren Alter vom ausgehenden Hochglazial bis tief in das Postglazial reicht. Die Bodenverhältnisse zeigen die talabwärts erfolgende Verjüngung der Oberflächen scharf an. Das Lechfeld - ein Teil der klassischen Niederterrasse, das etwa bei Hurlach beginnt und bis in die Gegend von Königsbrunn reicht - hat bodenkundlich mit den Terrassenstufen 1-6 (Niederterrassen, definiert durch Verknüpfung mit den äußeren Endmoränenwällen) nichts mehr gemein. Der geringe Verlehmungsgrad seiner Böden, im Gegensatz zu den Niederterrassenböden südlich Landsberg ist nicht in erster Linie eine Folge der abnehmenden Niederschlagsmengen, sondern der sehr viel später einsetzenden Bodenbildung. Das Nachhinken der Bodenentwicklung ist dadurch zu erklären, daß der Fluß das Lechfeld bis ins Postglazial noch als Hochwasserbett benutzte und damit die Bodenentwicklung hemmte, während die Niederterrassen südlich Landsberg schon mindestens seit dem frühen Spätglazial einer rein terrestrischen Bodenbildung unterlagen. 


\section{B. Die Böden der Lechterrassen}

\section{Die wichtigsten Faktoren der Bodenbildung}

a) Ausgangsmaterial

Das Ausgangsmaterial der Böden bilden hochkalkhaltige Schotter, die auf den jüngeren Terrassen von schluffigen bis schluffig-feinsandigen Sedimenten (Flußmergel) überdeckt werden. Da die jüngeren Schotter meist aus der Umlagerung der älteren entstanden sind, ist ihre Zusammensetzung über alle Terrassenstufen hinweg annähernd gleich.

1. Schotter

Die Korngrößenzusammensetzung der Schotter schwankt stark: Von Lagen mit weit über kopfgroßen Geröllen bis zum Sand finden sich alle Übergänge. Im allgemeinen überwiegen jedoch die Korngrößen unter $4 \mathrm{~cm}$ bei weitem. Nur in den älteren Terrassen mit starkem Gefälle $(>5 \%$ ) verschiebt sich das Verhältnis lagenweise zugunsten der gröberen Gerölle. Der Anteil der Fraktion $\langle 2 \mathrm{~mm}$ beträgt im Durchschnitt zwischen 25 und $40 \%$.

Der Gehalt an Kristallingeröllen schwankt aufgrund mehrerer Schotterauszählungen innerhalb der Schottergrößen von 6-40 mm zwischen 2 und 4\%. An frischen Schotterproben der Niederterrasse wurde versucht, auch den karbonatischen Anteil stärker zu gliedern. Demnach setzen sich die Lechschotter wie folgt zusammen:

\begin{tabular}{llr}
\hline Gesteinstyp & Herkunftsgebiet & Gewichtsanteil \% \\
\hline Kalkmergel und Mergelkalke & Flysch, Jura (Lias-Fleckenmergel, & \\
& Malm-Mergelkalke) & 25 \\
Dunkle, unreine Kalke & Muschelkalk, Raibler Kalk, Malm- und & 21 \\
Dolomite & Flyschkalke & 21 \\
Reine Kalke & vor allem Hauptdolomit & 13 \\
Kalksandsteine und Sandkalke & vor allem Wettersteinkalk & Molasse, Flysch (Reiselsberger Sandstein, Gault) \\
Hornsteine und Kieselkalke & Flysch, Jura & 13 \\
Kristalline Gerölle, Quarze & Zentralalpen & 5 \\
\hline
\end{tabular}

Zur Feststellung der Karbonatgehalte wurden 3 Proben zu je etwa 3,5 kg gequetscht, feingemahlen und nach der Komplexon-Methode auf ihre Kalzit- und Dolomitanteile untersucht. Die Proben stammen aus der jeweils obersten Terrassenstufe des Talabschnitts Epfach-Kaufering. Sie zeigen hinsichtlich der Karbonatgehalte und des Kalzit-DolomitVerhältnisses eine weitgehende Gleichartigkeit des Ausgangsmaterials (Tab. 1).

Tabelle 1

Karbonatgehalte und Kalzit-Dolomit-Verhältnis der Lechschot ter

\begin{tabular}{lcccc}
\hline Ort & Karbonatgehalt $\%$ & Kalzit & Dolomit & Kalzit/Dolomit \\
\hline Epfach & 73,2 & 33,6 & 39,6 & 0,85 \\
Pitzling & 74,4 & 33,8 & 40,6 & 0,83 \\
Kaufering & 74,5 & 36,2 & 38,3 & 0,94 \\
\hline Durchschnitt & 74,0 & 34,5 & 39,5 & 0,87
\end{tabular}

Die Karbonatgehalte und das Kalzit-Dolomit-Verhältnis unterliegen in den einzelnen Kornfraktionen großen Schwankungen. Im allgemeinen läßt sich beobachten, daß die Karbonat- und Kalzitgehalte in den feineren Fraktionen geringer werden, während der Dolomitgehalt weitgehend gleich bleibt. Zu ähnlichen Ergebnissen gelangte auch F. KoHL (1965) bei seiner Untersuchung des nichtkarbonatischen Anteils in südbayerischen Schottern. 
Tabelle 2

Karbonatgehalte und Kalzit-Dolomit-Verhältnis der Lechschotter in $A b h a ̈ n g i g k e$ it von der Korngröße

\begin{tabular}{lcccc}
\hline & Karbonatgehalt $\%$ & Kalzit & Dolomit & Kalzit/Dolomit \\
\hline Fraktion $>2 \mathrm{~mm}$ & 79,2 & 38,7 & 40,5 & 0,96 \\
Fraktion $<2 \mathrm{~mm}$ & 65,8 & 27,3 & 38,5 & 0,71
\end{tabular}

2. Flußmergel

Die Zusammensetzung des frischen Flußmergels kann nur noch auf den jüngsten Terrassen untersucht werden, da die geringmächtigen Flußmergelauflagen der älteren Terrassen restlos entkalkt und verwittert sind.

In Tabelle 3 ist die Korngrößenverteilung von 4 verschiedenen Flußmergelproben zusammengestellt. Sie zeigt eine eindeutige Vormacht der Mittelschluff- bis Feinsandfraktion.

Tabelle 3

Die Korngrößenverteilung des Flußmergels der jüngeren Lechterrassen

\begin{tabular}{|c|c|c|c|c|c|c|c|c|c|}
\hline \multirow[b]{2}{*}{ Ort } & \multirow[b]{2}{*}{$\begin{array}{l}\text { Labor- } \\
\text { Nr. }\end{array}$} & \multicolumn{7}{|c|}{ Korngrößen \% } & \multirow{2}{*}{$\begin{array}{c}\text { Bodenartl. } \\
\text { Gesamt- } \\
\text { charakter }\end{array}$} \\
\hline & & $\begin{array}{c}\mathrm{T} \\
<0.002 \\
\mathrm{~mm}\end{array}$ & $\mid \begin{array}{c}f U \\
0.002- \\
0.006\end{array}$ & \begin{tabular}{|l|}
$\mathrm{mU}$ \\
$0.006-$ \\
0.02
\end{tabular} & $\begin{array}{l}\mathrm{gU} \\
0.02- \\
0.06\end{array}$ & $\begin{array}{l}\text { fS } \\
0.06- \\
0.2\end{array}$ & $\begin{array}{l}\mathrm{mS} \\
0.6 \\
0.2-\end{array}$ & $\begin{array}{l}\text { gS } \\
0.6- \\
2.0\end{array}$ & \\
\hline \multirow{2}{*}{$\begin{array}{l}\text { südl. Lands- } \\
\text { berg (Pößin- } \\
\text { ger Au) }\end{array}$} & 23788 & 15,8 & 7,9 & 16,6 & 38,8 & 20,7 & 0,2 & - & slu \\
\hline & 23790 & 11,7 & 9,9 & 24,8 & 38,6 & 14,4 & 0,6 & - & l'U \\
\hline südl. & 25768 & 9,9 & 5,2 & 20,3 & 45,2 & 19,0 & 0,4 & - & l'U \\
\hline Kaufering & 25770 & 2,1 & 0,3 & 2,3 & 1,4 & 82,2 & 11,7 & - & fS \\
\hline
\end{tabular}

Um die Frage nach der Herkunft der Flußmergel zu klären, verglich M. SALGER ${ }^{1}$ ) die Mineralzusammensetzung der Flußmergel mit der des Flinzes und der schluffreichen Endmoräne. Nach dem Kalzit-Dolomitverhältnis sowie den Quarz-, Feldspat- und Glimmergehalten besteht der Flußmergel aus etwa gleichen Anteilen von Flinz- und Endmoränenmaterial. Der Karbonatgehalt schwankt etwa zwischen 60 und 70\% (Tab. 4). Rd. 80\% der Karbonate bestehen aus Dolomit. Die nichtkarbonatischen Minerale setzen sich in erster Linie aus Quarz, Glimmer, Feldspat, Hornblende, Biotit und Chlorit zusammen.

Tabelle 4

Karbonatgehalte und Kalzit-Dolomitverhältnis der Lech-F lu B mergel

\begin{tabular}{lccccc}
\hline Ort & $\begin{array}{c}\text { Labor- } \\
\text { Nr. }\end{array}$ & $\begin{array}{c}\text { Karbonat- } \\
\text { gehalt } \%\end{array}$ & $\begin{array}{c}\text { Kalzit } \\
\%\end{array}$ & $\begin{array}{c}\text { Dolomit } \\
\text { \% }\end{array}$ & $\begin{array}{c}\text { Kalzit/ } \\
\text { Dolomit }\end{array}$ \\
\hline südl. Landsberg & 23788 & 58,9 & 3,6 & 55,3 & 0,07 \\
(Pößinger Au) & 23790 & 66,1 & 13,2 & 52,9 & 0,25 \\
\hline südlich Kaufering & 25768 & 70,4 & 15,3 & 55,1 & 0,28 \\
\hline Durchschnitt & 25770 & 71,9 & 16,4 & 55,5 & 0,30 \\
\hline
\end{tabular}

1) ORR Dr. M. SALGER, Bayerisches Geologisches Landesamt, München, unveröffentlichtes Manuskript. 
b) K 1 i m a

Die für die Bodenentwicklung wesentlichen klimatischen Verhältnisse des Untersuchungsgebietes sind nach dem Klimaatlas von Bayern in der nachstehenden Übersicht zusammengestellt:

\begin{tabular}{|c|c|c|c|c|}
\hline & Schongau & Lechblick & Landsberg & Lager Lechfeld \\
\hline $\begin{array}{l}\text { Mittlere wirkliche Luftemperatur }{ }^{\circ} \mathrm{C} / \mathrm{J} \\
(1881-1930) \\
\text { Mittlere wirkliche Luftemperatur }{ }^{\circ} \mathrm{C} \text { in der }\end{array}$ & 6,5 & 6,8 & 7,0 & 7,2 \\
\hline $\begin{array}{l}\text { Mittlere wirkliche Luttemperatur } \mathrm{C} \text { in der } \\
\text { Vegetationsperiode (Mai-Juli) } \\
\text { Mittlere Niederschlagssummen } \mathrm{mm} / \mathrm{Jahr}\end{array}$ & 13, & 14,0 & 14,3 & 14,5 \\
\hline $\begin{array}{l}(1881-1930) \\
\text { Mittlere Niederschlagssummen } \mathrm{mm} \text { in der }\end{array}$ & 1100 & 1000 & 950 & 800 \\
\hline Vegetationsperiode (Mai-Juli) & 450 & 380 & 350 & 300 \\
\hline
\end{tabular}

Die Niederschlagsmenge zeigt von Lager Lechfeld bis Schongau eine Zunahme von etwa $100 \mathrm{~mm}$ pro $14 \mathrm{~km}$ Entfernung. Nach J. van EIMERN (1967) kann die Jahresregenmenge sowohl bis zu $1 / 3$ größer als auch kleiner als normal sein. Die Monatsmittel können in einzelnen Jahren das Doppelte des Normalen betragen, während umgekehrt, wenn auch seltener, Trockenperioden von 4 Wochen Dauer möglich sind. Die Höhenlage (rd. $550-730 \mathrm{~m}$ über NN) bedingt bei wolkenarmem Wetter, insbesondere unter dem Einfluß des Föhns, eine sehr intensive Einstrahlung.

c) Vegetation und Nutzung

Die ursprünglichen Eichen-Mischwälder der grundwasserfreien Lech-Terrassenlandschaft sind heute nirgends mehr erhalten. Soweit überhaupt noch Wald vorhanden ist, handelt es sich größtenteils um standortsuntypische Fichtenbestände. Nur die jüngsten Auenstufen tragen noch eine weitgehend natürliche Auenwaldvegetation.

Der weitaus größte Teil der Terrassenlandschaft wird heute landwirtschaftlich genutzt. Der Verlauf der Römerstraße Via Claudia Augusta auf den älteren Terrassenstufen sowie zahlreiche, auf die germanische Landnahme im 5. und 6. nachchristlichen Jahrhundert zurückgehende Ortsgründungen am westlichen Talrand (Ortsnamen mit der Endung ,ing“) lassen vermuten, daß zumindest Teile der Terrassenflächen schon seit dem frühen Mittelalter oder noch länger landwirtschaftlich genutzt werden.

Die Beackerung hatte auf den von Natur aus sehr flachgründigen Böden eine um so einschneidendere Wirkung auf die Bodenentwicklung, je länger sie betrieben wurde und je geringmächtiger das Solum ausgebildet war. $\mathrm{Zu}$ der mischenden Wirkung des Pfluges, der die ursprünglichen oberen Bodenhorizonte zum Opfer fielen, gesellte sich ein langsamer, im Lauf vieler Jahrhunderte jedoch recht beträchtlicher Substanzschwund (s. S. 117), der zur Folge hatte, daß allmählich auch die unteren Bodenhorizonte in den Pflugbereich gelangten und schließlich sogar kalkhaltiges Untergrundmaterial heraufgepflügt wurde. Auf diesen Umstand ist die Beobachtung zurückzuführen, daß sich die landwirtschaftlich genutzten Böden auch von zeitlich weit auseinanderliegenden Terrassen heute weitgehend gleichen.

\section{Morphologie, Genese und Eigenschaften der Böden der einzelnen Terrassen- stufen}

Der Beginn des Eisrückzuges von den äußersten Würm-Endmoränenwällen bedeutet gleichzeitig den Beginn der Bodenbildung auf der unmittelbar mit der Endmoräne verknüptten Hauptniederterrasse. In gleicher Weise bedeutet jedes Einschneiden des Flusses in sein zuvor benutztes Bett den Beginn der Bodenbildung auf der damit neu entstandenen Terrassenstufe. Das Alter der Böden nimmt daher vor der obersten zur untersten Terrasse eines Talquerschnittes ab. 
Komplizierter liegen die bodenkundlichen Verhältnisse im Tallängsschnitt. Darauf wurde bereits im vorhergehenden Kapitel hingewiesen.

Will man das Ergebnis der Bodenbildungsprozesse auf den einzelnen Terrassen als Funktion der Zeit erfassen, so ist es unerläßlich, nach Bodenformen zu suchen, die weder durch natürliche Erosion oder Akkumulation noch durch menschliche Eingriffe in ihrem Profilbild wesentlich verändert wurden. Solche Bodenformen sind - wenigstens auf den älteren Lechterrassen - i. a. nur unter Wald anzutreffen. Sie repräsentieren gleichsam die $\mathrm{Maximalformen} \mathrm{der} \mathrm{innerhalb} \mathrm{einer} \mathrm{gegebenen} \mathrm{Zeitspanne} \mathrm{möglichen} \mathrm{Boden-}$ entwicklung. Aus ihrer Kenntnis lassen sich die Veränderungen der Böden unter dem Einfluß der Ackerkultur abschätzen sowie Kriterien für die Unterscheidung der auf den ersten Blick fast gleichartig erscheinenden landwirtschaftlich genutzten Böden verschiedener Terrassen erarbeiten. Die nachstehenden Ausführungen über die Bodenbildungen der einzelnen Terrassenstufen beziehen sich in erster Linie auf die Maximalformen, auch wenn sie teilweise nur sehr wenig flächenrepräsentativ sind.

Zur Eigenart der Böden aus hochkarbonathaltigem Ausgangsmaterial gehört eine geringe Entwicklungstiefe und damit die Konzentration der verschiedenen bodenbildenden Prozesse auf eine geringmächtige Verwitterungsschicht. Die für die Einordnung der Böden in das genetische System relevanten Bodenhorizonte sind häufig nur wenige Zentimeter mächtig. Ihre Erkennung erfordert höchste Sorgfalt bei Profiluntersuchungen und der Probenentnahme im Gelände.

a) Die Böden der ältesten Lechterrassen (= Niederterrassen, Stuf en 1-6)

Die Niederterrassen, zu denen die Hauptniederterrasse und die Stufen von St. Ursula, Altenstadt (Römerau), Hohenfurch, Schongau-Peiting und Unterigling gerechnet werden, tragen weitgehend ähnliche, höchstens in der Mächtigkeit ihres Solums etwas differierende Böden. Es handelt sich um Parabraunerden von durchschnittlich 4-5 dm Entwicklungstiefe mit auffallend rötlichbraun gefärbtem $\mathrm{B}_{\mathrm{t}}$-Horizont, die in der Literatur als „Blutlehm", „Kreßlehm“ und „Schotterlehm“ bezeichnet wurden (Literaturangaben s. S. 124).

Die nachstehend beschriebenen Profile 1 und 2 veranschaulichen den für die LechNiederterrassen unter Wald typischen Profilaufbau.

\section{Profil 1: $\quad$ R $13300 \quad$ H 29150}

Kaufering Bhf NW; Terrasse; Wald (Fichte, Kiefer)

$\mathrm{P}$ a r a b r a u e r d e mittlerer Entwicklungstiefe aus Schotter

$\mathrm{O}_{\mathrm{L}, \mathrm{F}, \mathrm{H}} \quad 2 \mathrm{~cm}$ Moder

$\mathrm{A}_{\mathrm{h}}$ 0-10 cm braungrauer, humoser, schwach steiniger, feinsandiger Lehm; subpoly-

$A_{1} \quad 10-28 \mathrm{~cm}$ gelbbrauner, steiniger, feinsandiger, schwach toniger Lehm; subpoly-

edrisches Gefüge

$\mathrm{B}_{\mathrm{t}} \quad 28-35 \mathrm{~cm} \quad$ rötlichbrauner, steiniger, feinsandig-toniger Lehm; polyedrisches Gefüge;

$\mathrm{C}_{\mathrm{n}} \quad 35-50 \mathrm{~cm}+$ einzelne Kalks Kalks

Profil 2: $\quad$ R 16840 H 07210

Epfach SW; Kiesgrube an der Bundesstraße 17, Abzweigung Epfach; Acker

P a r a b r a u n e d e mittlerer Entwicklungstiefe aus Schotter

$A_{p} \quad 0-16 \mathrm{~cm}$ dunkelbraungrauer, humoser, schwach steiniger, feinsandiger Lehm, sub-

A $16-32 \mathrm{~cm} \quad$ polyedrisches Gefüge

$A_{1} \quad 16-32 \mathrm{~cm}$ rötlichgelbbrauner (trocken graugelber), steiniger, feinsandiger, schwach

$\mathrm{B}_{\mathrm{t}} \quad 32-43 \mathrm{~cm} \quad \begin{aligned} & \text { toniger Lehm; subpolyedrisches Gefüge } \\ & \text { rötlichbrauner, steiniger, feinsandig-toniger Lehm; polyedrisches Gefüge, }\end{aligned}$

rotbraune Tonhäutchen; einzelne Kalkgerölle

$\mathrm{C}_{\mathrm{n}} \quad 43-100 \mathrm{~cm}+$ sandiger Fein- und Grobkies.

Analysen vgl. Tab. 5. 
Die mit 4-5 dm angegebene durchschnittliche Entwicklungstiefe wird häufig durch taschen- oder zapfenartige Einstülpungen überschritten. Die unregelmäßige, gegen den kalkreichen Schotteruntergrund scharf abgesetzte Untergrenze des $\mathrm{B}_{\mathrm{t}}$-Horizontes ist teilweise auf schwache Kryoturbation, teilweise auf Zonen bevorzugter Sickerwasserbewegung (Körnungsunterschiede, Wurzeln) und damit verstärkter Lösungsverwitterung zurückzuführen. Der starke Steingehalt der Verwitterungshorizonte besteht aus nichtkarbonatischen, meist scharfkantig-grusig zerfallenden Restgeröllen und stellt das pedologische Hauptunterscheidungsmerkmal zwischen den älteren und jüngeren Lechterrassen dar.

Unter Wald sind die Eluvialhorizonte unter einer geringmächtigen Moder- bis Rohhumusauflage stark bis sehr stark sauer und besitzen nur eine geringe Basensättigung. Im $\mathrm{B}_{\mathrm{t}}$-Horizont steigen $\mathrm{pH}$-Wert und Basensättigung sprunghaft an und nähern sich den Werten des hochkalkhaltigen Untergrundes. Durch die Ton- und Eisenverteilung im Profil wird die Durchschlämmung ebenso angezeigt, wie durch das makro- und mikromorphologische Profilbild.

Bemerkenswert ist die Gleichmäßigkeit der Bodenbildung von Kaufering bis Schongau, d. h. über eine Strecke von rd. $30 \mathrm{~km}$ auf der die Niederschläge von $900 \mathrm{~mm}$ bis auf $1100 \mathrm{~mm}$ zunehmen. Selbst die durchschnittliche maximale Entwicklungstiefe ändert sich nur wenig. Sie dürfte in Höhe von Schongau näher bei 5, in Höhe von Kaufering näher bei $4 \mathrm{dm}$ liegen.

Nach K. Brunnacker (1957, S. 63) soll die Entwicklungstiefe der „Schotterlehme“ aus IsarNiederterrassenschottern bei etwa gleicher Zunahme der Niederschlagsmenge von $35 \mathrm{~cm}$ auf $70-80 \mathrm{~cm}$ ansteigen. Diese etwas widersprüchlichen Angaben über die Zusammenhänge zwischen Klima und Bodenbildung dürften zum einen auf der Schwierigkeit der Ermittlung durchschnittlicher Entwicklungstiefen beruhen (zuverlässige Durchschnittswerte sind praktisch nur durch eine Kartierung alter Waldflächen oder durch große Aufschlüsse in morphologisch geeigneter Geländeposition zu erhalten), zum anderen ist die Gleichheit des Alters bzw. der spätglazial wirksamen Grundwasserverhältnisse der von K. BRUNNACKER verglichenen Bodenbildungen in Zweifel zu ziehen.

Zum Vergleich seien die vom Verfasser beobachteten, durchschnittlichen Entwicklungstiefen der entsprechenden Bodenbildungen auf Niederterrassenschottern der östlich gelegenen Alpenflüsse angegeben: Sie betragen auf der Schotterebene südlich München um $5 \mathrm{dm}$, am Inn bei Gars (PipelineAufschluß) 6-7 dm. Die größeren Entwicklungstiefen am Inn sind auf den wesentlich größeren Nichtkarbonatanteil der Schotter (er beträgt nach F. KoHL [1965] ca. 60\%) zurückzuführen.

Die in den Profilen 1 und 2 gezeigte Maximalbodenbildung der oberen Lechterrassen wird auf den landwirtschaftlich genutzten Flächen weithin von einer weniger mächtigen Bodenbildung vertreten; sie gleicht auf den ersten Blick einer Rendzina. Die Mächtigkeit des Solums ist auf 2,5-3,5 dm zusammengeschrumpft. Die humose Krume liegt häufig dem Kalkschotter direkt auf. Starke Restgeröllanreicherung, ein leicht rötlicher Farbton der Krume und ein an der Schottergrenze teilweise noch erkennbarer rötlichbrauner Saum oder in den Schotter hineinragende Verwitterungszapfen zeigen jedoch an, daß die Bodenform aus der oben beschriebenen Parabraunerde hervorgegangen ist. Sie wurde auf Blatt Landsberg analog zu ähnlich entstandenen Böden aus Löß (I. LIEBEROTH 1962, K. BRUNNACKer 1963, S. 47) als „Ackerbraunerde" kartiert. Profil 3, auf einer den Lech-Niederterrassen entsprechenden Schotterfläche östlich Landsberg gelegen, ist ein Beispiel für eine solche Ackerbraunerde.

Profil 3: R 20380 H 20350

Kiesgrube Pürgen SE; Schotterflur, eben; Grünland

A c k e r b r a u n e d e mittlerer Entwicklungstiefe aus Schotter

$A_{p} \quad 0-25 \mathrm{~cm}$ dunkelgraubrauner, sehr stark humoser, steiniger, feinsandiger Lehm; bröckelig-krümeliges Gefüge

$\mathrm{AB} \quad 25-35 \mathrm{~cm}$ dunkelbrauner, stark humoser, steiniger, sandiger, schwach toniger Lehm;

feinsubpolyedrisches Gefüge

$\mathrm{C}_{\mathrm{v}} \quad 35-40 \mathrm{~cm}+$ sandiger Kies (Kalkschotter) mit humosem, tonig-lehmigem Zwischen-

Analysen vgl. Tab. 5. mittel. 
Die chemischen Analysendaten der Ackerbraunerde zeigen kaum noch Ähnlichkeit zu denen der Parabraunerde, aus der sie hervorging. Sie besitzt neutrale Reaktion, hohe Basensättigung und ein enges $\mathrm{C} / \mathrm{N}-$ Verhältnis im ganzen Profil. An die ursprüngliche Parabraunerde erinnert nur noch der gegen die Untergrenze des Solums stark ansteigende Gehalt an dithionitlösl. Eisen (der jedoch auch fehlen kann).

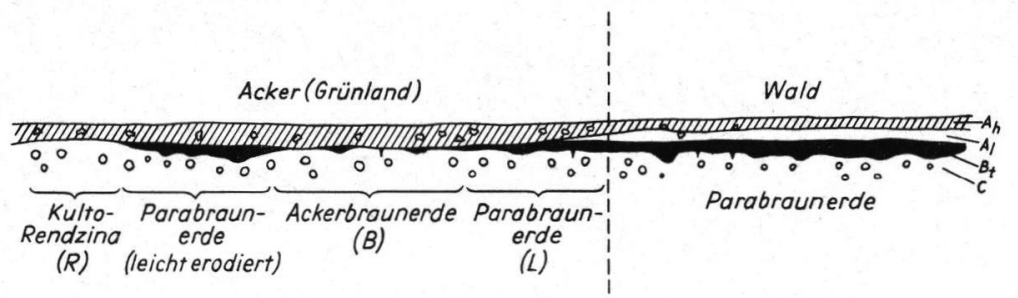

Abb. 5. Die Bodenvergesellschaftung auf den Niederterrassen in Abhängigkeit von der Bodennutzung.

Abb. 5 veranschaulicht die unterschiedliche Ausbildung der Niederterrassenböden in Abhängigkeit von der Nutzung. Der gegenüber den Waldböden an der geringeren Entwicklungstiefe feststellbare Substanzschwund ist, da Abspülung wegen des ebenen Reliefs nicht in Frage kommt (eine flächenhafte Überflutung ist nach der glazialen Schmelzwasserphase nicht mehr denkbar), nur durch Winderosion zu erklären.

Würde das Profilbild nicht eine so deutliche Sprache sprechen, so hielte man eine Winderosion unter den gegebenen Klimabedingungen (950-1100 mm Niederschlag!) kaum für möglich. Stellt man dagegen in Rechnung, daß die Böden infolge ihres geringmächtigen Solums und ihres durchlässigen Untergrundes mitunter stark austrocknen können, so erscheint ein äolischer Bodenabtrag auf den kilometerbreiten, waldlosen Terrassenflächen (=Windangriffsflächen) unter Ackernutzung während der vegetationsfreien Zeit immerhin denkbar. Bei einer nach der Besiedlungsgeschichte durchaus möglichen, über tausendjährigen Ackerkultur (vgl. S. 114) würde bereits $1 / 10 \mathrm{~mm}$ Abtrag pro Jahr genügen, um die beobachtete Differenz in der Mächtigkeit des Solums zwischen Ackerund Waldböden zu bewirken. Profilmorphologisch ist der Unterschied zwischen Acker- und Waldböden nur deshalb so augenfällig, weil es sich schon primär um einen nur geringmächtigen Boden handelte und der hohe Kalkgehalt des Ausgangsmaterials eine Kompensation des Bodenabtrags durch eine Tiefenentwicklung vergleichbaren Ausmaßes verhindert. Die Verarmung des Solums (auch des Unterbodens) an Feinboden ist durch die mischende Wirkung des Pfluges und selektive Erosion zu erklären (G. Richter 1965, S. 168 ff.).

Nach mündlicher Mitteilung von Herrn Oberregierungslandwirtschaftsrat EGER/Schwabmünchen ist das Gebiet der Lechterrassen ebenso wie das der westlich anschließenden Lößplatte außerordentlich starken Winden ausgesetzt. Gegen Ende des vorigen Jahrhunderts wurden deshalb auf der Niederterrasse von dem damaligen Bewirtschafter Lichti Windschutzpflanzungen angelegt, die heute allerdings nur noch in Resten erhalten sind.

Lokal geht der Sustanzverlust auf den landwirtschaftlich genutzten Flächen so weit, daß der Pflug heute schon frisches Kalkschottermaterial an die Oberfläche bringt. Derartige Flächen besitzen ein reines A-C-Profil mit einer Mächtigkeit des Solums von weniger als 2,5 dm. Der A-Horizont ist sekundär aufgekalkt. Im Gegensatz zu jungen Mullrendzinen und gleichzeitig als Merkmale für ihre Entstehung aus der oben beschriebenen Parabraunerde besitzen diese Böden einen mit nichtkarbonatischen Restgeröllen stark angereicherten, schwach rötlich getönten $A_{p}$-Horizont. Sie werden, da allein durch Ackerkultur entstanden, als Kulto-Rendzinen bezeichnet. Analoge Bodenbildungen aus den weniger karbonatreichen Flußsedimenten der Isar wurden von K. BRUNNACKER (1960) als "Regradierte Pararendzinen" angesprochen.

b) Die Böden der Terrassenstufe von Friedheim (8)

Zwischen den Böden der Lech-Niederterrassen und denen der Friedheimer Terrasse liegt ein erheblicher Entwicklungsunterschied, wie eine Gegenüberstellung der Profile 1 und 2 mit Profil 4, der Maximalbodenbildung der Terrasse von Friedheim, zeigt. Die Entwicklungstiefe des kalkfreien Maximalbodens beträgt nur noch knapp die Hälfte, die Gesamt- 
menge an nichtkarbonatischen Lösungsrückständen ist dementsprechend geringer. Vor allem fehlt der für die Parabraunerde so charakteristische, rötlichbraune, tonig-lehmige $\mathrm{B}_{\mathrm{t}}$-Horizont.

Profil 4 :

R 14830 H 19460

Ellighofen NE; Terrasse; Wald (Kiefer mit dichtem Unterwuchs von Brachypodium pinnatum)

Braunerde geringer Entwicklungstiefe aus Schotter mit beginnender Tondurchschlämmung

\begin{tabular}{|c|c|c|}
\hline $\begin{array}{l}\mathrm{O}_{L, F} \\
\mathrm{~A}_{\mathrm{h}}\end{array}$ & $\begin{array}{r}3 \mathrm{~cm} \\
0-5 \mathrm{~cm}\end{array}$ & $\begin{array}{l}\text { Graswurzelfilz mit verrottenden Kiefernnadeln } \\
\text { graubrauner, sehr stark humoser, schwach steiniger, schwach toniger Lehm; } \\
\text { feinsubpolyedrisches Gefüge }\end{array}$ \\
\hline $\mathrm{B}_{\mathrm{v} 1}$ & $5-10 \mathrm{~cm}$ & $\begin{array}{l}\text { gelbgraubrauner, schwach steiniger, schwach toniger Lehm; feinsubpoly- } \\
\text { edrisches Gefüge }\end{array}$ \\
\hline $\mathrm{B}_{\mathrm{v} 2}$ & $10-15 \mathrm{~cm}$ & $\begin{array}{l}\text { gelbgraubrauner, schwach steiniger, toniger Lehm; mittelsubpolyedrisches } \\
\text { Gefüge }\end{array}$ \\
\hline$B C$ & $15-30 \mathrm{~cm}$ & $\begin{array}{l}\text { Kalkschotter mit gelbbraunem, weitgehend kalkfreiem, sandig-lehmigem } \\
\text { Zwischenmittel. }\end{array}$ \\
\hline
\end{tabular}

Analysen vgl. Tab. 5.

Daß die Entwicklungstendenz zur Parabraunerde geht, ist bereits im Gelände auf Grund der Gefügeunterschiede zu vermuten und wird durch die Zunahme der Ton- und DeB-Eisengehalte vom $A_{h}$ - zum $B_{v 1}$ - und $B_{v 2}$-Horizont bestätigt. Bei insgesamt nur $15 \mathrm{~cm}$ Solum sind die oberen Bodenhorizonte bereits stark bis sehr stark sauer, die Basensättigung ist mittel bis gering. Die Flora des Standorts läßt darauf schließen, daß es sich um eine alte Waldweide handelt. Dies würde auch das relativ enge $\mathrm{C} / \mathrm{N}-$ Verhältnis erklären.

Von den Böden der jüngeren Terrassen unterscheiden sich die Böden der Friedheimer Terrasse vor allem durch die Anreicherung des Solums mit nichtkarbonatischen Restgeröllen und die kräftig gelbbraune Farbe der $B_{\mathrm{v}}$-Horizonte. Der weitaus größte Teil der Terrasse wird landwirtschaftlich genutzt und zeigt dann das durch die homogenisierende Wirkung des Pfluges herbeigeführte $\mathrm{A}_{\mathrm{p}}$-C-Profil der Kulto-Rendzina. Farbe und Restgeröllgehalt sind auch in diesem Entwicklungsstadium noch brauchbare Unterscheidungskriterien zur Abgrenzung gegenüber den Böden der jüngeren Terrassenstufen.

c) Die Böden der Terrassenstufe von Kaufering Bhf(9)

und vom Z e h nerhof (10)

Obwohl die nur morphologisch gegeneinander abgrenzbaren Terrassen eindeutig jünger sind als die Terrassenstufe von Friedheim, erscheinen ihre Maximalbodenbildungen typologisch älter. Der Grund dafür liegt darin, daß die hier erstmalig auftretenden und für alle jüngeren Terrassen kennzeichnenden Flußmergelauflagen rascher verwittern als die karbonatreicheren, mechanisch noch nicht so weit aufbereiteten Schotter.

Profil 5 zeigt etwa die Maximalbodenbildung dieser Terrassenstufen, eine Braunerde mit deutlicher Tondurchschlämmung, wie sie nur dort vorkommt, wo die Flußmergeldecke eine Mächtigkeit von $2 \mathrm{dm}$ oder mehr erreicht ${ }^{2}$ ). Beträgt die Flußmergelauflage nur etwa $1 \mathrm{dm}$, so geht die Bodenentwicklung nicht über das Stadium der Rendzina-Braunerde hinaus (Profil 6).

Profil 5: $\quad$ R 15610 H 20370

Lech-Staustufe 14 NW; Terrasse; Wald (Fichte, etwas Kiefer)

$\mathrm{B} r$ a u n e $\mathrm{d}$ e geringer Entwicklungstiefe mit Tondurchschlämmung aus Flußmergel über Schotter

2) Die Mächtigkeitsangaben für die Flußmergelauflage beziehen sich auf die aus dem Flußmergel hervorgegangenen Bodenhorizonte ohne Berücksichtigung des durch die Karbonatauswaschung eingetretenen Substanzverlustes. Die aus Flußmergel entstandenen Bodenhorizonte unterscheiden sich im ungestörten Profil von den Schotterverwitterungshorizonten durch das Fehlen von nichtkarbonatischen Restgeröllen. 


\begin{tabular}{|c|c|c|}
\hline $\begin{array}{l}\mathrm{O}_{L, F}, \mathrm{H} \\
\mathrm{A}_{\mathrm{h}}\end{array}$ & $\begin{array}{r}4 \mathrm{~cm} \\
0-5 \mathrm{~cm}\end{array}$ & $\begin{array}{l}\text { rohhumusartiger Moder } \\
\text { braungrauer, stark humoser, schwach toniger Lehm; feinsubpolyedrisches }\end{array}$ \\
\hline$A_{1} B_{V}$ & $5-15 \mathrm{~cm}$ & $\begin{array}{l}\text { gelbbraungrauer, humoser, schwach toniger Lehm; mittel- bis feinsubpoly- } \\
\text { edrisches Gefüge }\end{array}$ \\
\hline$B_{t^{v}}$ & $15-22 \mathrm{~cm}$ & $\begin{array}{l}\text { gelbbrauner, toniger Lehm; mittelpolyedrisches Gefüge; an Untergrenze } \\
\text { Anreicherung von Restgeröllen }\end{array}$ \\
\hline $\begin{array}{l}\text { IIBC } \\
\mathrm{C}_{\mathrm{n}}\end{array}$ & $\begin{array}{l}22-45 \mathrm{~cm} \\
45-50 \mathrm{~cm}+\end{array}$ & $\begin{array}{l}\text { Kalkschotter mit } \mathrm{B}_{\mathbf{t}}-\mathrm{Z} \text { wischenmittel } \\
\text { sandiger Kies. }\end{array}$ \\
\hline
\end{tabular}

Profil 6: R 15580 H 19850

Lech-Staustufe 14 WNW; Terrasse; Wald (Kiefer, Fichte)

$\mathrm{Rendz}$ in a-B ra un erde mit rohhumusartiger Moderauflage aus Flußmergel über Schotter

$\mathrm{O}_{\mathrm{L}, \mathrm{F}, \mathrm{H}} \quad 3 \mathrm{~cm} \quad$ rohhumusartiger Moder

$\mathrm{A}_{\mathrm{h}}$ O- $5 \mathrm{~cm}$ dunkelgraubrauner, stark humoser, schluffiger, schwach toniger Lehm; feinsubpolyedrisches bis krümeliges Gefüge

$A_{h} B_{v} \quad 5-10 \mathrm{~cm}$ gelbgraubrauner, stark humoser, schwach toniger Lehm; mittel- bis fein-

subpolyedrisches Gefüge; an der Untergrenze Restgeröllanreicherung

IIBC $10-20 \mathrm{~cm}$ stark angewitterter Kalkschotter mit graubraunem, schwach humosem,

Analysen vgl. Tab. 5. schluffig-tonigem $\mathrm{Z}$ wischenmittel.

Ein Vergleich der Eisen-, Ton- und Basensättigungswerte zeigt, daß Verwitterung, Entbasung und Tonverlagerung in Profil 5 nicht die Intensität der Niederterrassen-Parabraunerden erreichen.

Angesichts des hohen Karbonatgehaltes des Ausgangsmaterials und der geringen Entwicklungstiefe der Böden überraschen besonders die rohhumusähnlichen organischen Auflagen. Sie dürften einerseits auf die nichtstandortsgemäße Fichtenbestockung, zum anderen auf die den Humusabbau hemmende ökologische Trockenheit der Böden zurückzuführen sein. Eine Parallele hierzu bilden die von P. Seybert (1958) in der Pupplinger Au beschriebenen „Tangel-Borowinen“ aus Isar-Schottern ${ }^{3}$ ).

Die landwirtschaftlich genutzten Böden sind ausschließlich Kulto-Rendzinen, die - im Gegensatz zu den Kulto-Rendzinen der älteren Terrassen - in der Krume nur vereinzelt Restgerölle führen. Ihre Farbe ist etwas dunkler. Sie sind entweder kalkfrei oder sekundär schwach aufgekalkt.

Nordwestlich von Landsberg finden sich auf dieser Terrassenstufe alte Hochäcker als Zeichen des Bemühens, die flachgründigen, trockenen Böden durch Zusammenpflügen der Krume in ihrer Ertragsfähigkeit zu verbessern.

d) Die Böden der Oberen (11) und Unteren Epfachstufe (12)

Die von K. Brunnacker (1964) so benannten, nur zwischen Kinsau und Epfach unterscheidbaren Terrassenstufen, sind denen der Stufen von Kaufering Bhf und vom Zehnerhof hinsichtlich ihrer Bodenbildungen ähnlich. Unter Wald kommt als Maximalbodenbildung aus etwa $2 \mathrm{dm}$ mächtigen Flußmergelauflagen eine Braunerde mit schwacher Tondurchschlämmung vor, die in ihrer Entwicklung ungefähr in der Mitte zwischen Profil 5 und 8 steht.

Die verbreitetste Bodenbildung dieser fast ausschließlich landwirtschaftlich genutzten Terrassenstufen ist eine Mullrendzina mit entkalktem oder sekundär schwach aufgekalktem A-Horizont. Die Bodenfarbe ist dunkler, die Gefügeaggregation etwas geringer als die der Kulto-Rendzinen der älteren Terrassen. Nichtkarbonatische Restgerölle fehlen weitgehend.

3) S. MÜLLER (mündl. Mitteilung) führt die Entstehung solcher saurer Auflagehumushorizonte auf Oberbodenstörung zurück. Die biologische Oberbodenstörung kann z. B. dann auftreten, wenn Böden, die unter landwirtschaftlicher Nutzung stark verarmt sind, mit Fichte aufgeforstet werden. 


\section{Profil 7: $\quad$ R $15360 \quad$ H 28280}

Kaufering Bhf NE; Terrasse; Grünland

$\mathrm{Mu} 11 \mathrm{r}$ end z in a mit entkalktem A-Horizont aus Flußmergel über Schotter

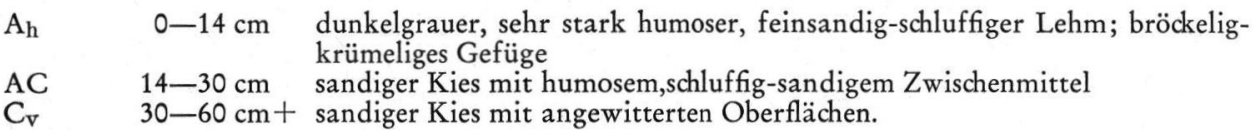

Analysen vgl. Tab. 5.

Genau genommen, stellen auch die Mullrendzinen dieser Stufe zumindest teilweise KultoRendzinen dar. Da sie sich jedoch vom Maximalstadium der Bodenentwicklung unter Wald um so weniger unterscheiden, je jünger die Terrassen werden und weil bei geringmächtigen Flußmergelauflagen auch die Maximalbodenbildung kaum über das Rendzinastadium hinausging, wird die Bezeichnung „Kulto-Rendzina“ nicht mehr verwendet.

Interessant ist ein Aufschluß bei Lager Lechfeld, in einem Talabschnitt, in dem sich die Epfacher Terrassen bereits mit den Niederterrassen gekreuzt haben. Aus einer etwa $1 \mathrm{dm}$ mächtigen Flußmergelauflage hat sich eine noch schwach kalkhaltige Mullrendzina entwickelt. Die Bodenbildung liegt teilweise dem frischen Kalkschotter direkt auf, teilweise ist ein bis zu $2 \mathrm{dm}$ mächtiger, dichtgepackter Restgeröllhorizont zwischengeschaltet, der als umgelagerter Rest der Niederterrassen-Bodenbildung zu deuten ist. Damit ist ungefähr die alte Oberfläche der jüngeren Niederterrasse markiert.

e) Die Böden der Oberen (13) und Unteren Lorenzbergstufe (14)

Die maximale Bodenbildung der Oberen Lorenzbergstufe (die Untere Lorenzbergstufe läuft bereits vor Pitzling aus; sie wird ausschließlich landwirtschaftlich genutzt) ist unter Wald nur noch auf Blatt Landsberg erhalten.

Profil 8: $\quad$ R $15880 \quad$ H 20170

Lech-Staustufe 14 NW; Terrasse; Wald (Fichte, Kiefer)

B r a u n e r d e geringer Entwicklungstiefe aus Flußmergel über Schotter

$\mathrm{O}_{\mathrm{L}, \mathrm{F}, \mathrm{H}} \quad 2 \mathrm{~cm}$ schlechter Moder

$\mathrm{A}_{\mathrm{h}}$ O- $4 \mathrm{~cm}$ graubrauner, stark humoser, schwach toniger Lehm; feinsubpolyedrisches

$\mathrm{B}_{\mathrm{v} 1} \quad 4-11 \mathrm{~cm}$ gelbgraubrauner, humoser, schwach toniger Lehm; feinsubpolyedrisches

$\mathrm{B}_{\mathrm{v} 2} \quad 11-18 \mathrm{~cm} \quad \begin{aligned} & \text { Gefüge } \\ & \text { gelbgraubrauner, humoser, toniger Lehm; fein- bis mittelsubpolyedrisches }\end{aligned}$ Gefüge; an der Basis Anreicherung von Restgeröllen.

Analysen vgl. Tab. 5.

Hinsichtlich Flußmergelauflage und Vegetation der Maximalbodenbildung der Stufe vom Zehnerhof (Profil 5) sehr ähnlich, zeigt Profil 8 an Eisengehalten, pH-Werten und $\mathrm{C} / \mathrm{N}-$ Verhältnis eine etwas geringere Verwitterungsintensität, kaum Tondurchschlämmung, eine weniger starke Bodenversauerung und eine etwas günstigere Humusform.

Die Normalbodenbildung auf landwirtschaftlich genutzten Flächen, eine dunkelgraue Mullrendzina mit entkalktem oder sekundär schwach aufgekalktem A-Horizont, ist von den Bodenbildungen der Epfachstufen unter gleicher Nutzung kaum zu unterscheiden.

f) Die Böden der Alteren Auenstufe (15)

Auf Grund der archäologischen Untersuchungen am Lorenzberg bei Epfach (S. 110) ergab sich für die Ältere Auenstufe ein maximales Alter von etwa 1600 Jahren. Das sehr junge Entwicklungsstadium der Böden und die geringe Höhendifferenz zum Normalflußwasserspiegel (ca. $3 \mathrm{~m}$ ) lassen allerdings vermuten, daß die Bodenbildung durch wiederholte Ưberflutung (Aufkalkung, evtl. Erosion) bis in jüngere Zeit in ihrer Entwicklung gehemmt wurde. 
Profil 9, am Fuß des Lorenzberges gelegen, stellt die Normalbodenbildung unter landwirtschaftlicher Nutzung, Profil 10 die Maximalbodenbildung unter Fichtenbestockung dar.

Profil 9: R 18960 H 08900

St. Lorenz SW; eben; Grünland

Rendzina a r tiger A u e n boden aus Flußmergel

$\mathrm{A}_{\mathrm{h}} \quad 0-25 \mathrm{~cm}$ bräunlichgrauer, sehr stark kalkhaltiger, feinsandig-lehmiger Schluff; sub-

polyedrisch-krümeliges Gefüge (schwache Gefügeaggregation)

AC $\quad 25-35 \mathrm{~cm}$ grauer, sehr stark kalkhaltiger, feinsandiger, schwach lehmiger Schluff

$\mathrm{C}_{\mathrm{n}} \quad 35-90 \mathrm{~cm}+$ gelbgrauer, feinsandiger Schluff bis schluffiger Feinsand.

Profil 10: R 16250 H 27130

Kaufering S; eben; Wald (Fichte)

Rendzina a r iger A u enboden (Borowina) aus Flußmergel, im Untergrund vergleyt

$\mathrm{O}_{\mathrm{L}, \mathrm{F}, \mathrm{H}} \quad 3 \mathrm{~cm} \quad$ typischer Moder

$A_{h 1} \quad 0-5 \mathrm{~cm}$ dunkelbraungrauer, sehr stark kalk- und humushaltiger, feinsandiger

Schluff; krümeliges Gefüge; Regenwürmer (bis in den AC) vorhanden

$\mathrm{A}_{\mathrm{h} 2} \quad 5-10 \mathrm{~cm}$ braungrauer, sehr stark kalk- und humushaltiger, feinsandiger Schluff;

krümeliges Gefüge

AC $10-15 \mathrm{~cm}$ braungrauer und graugelber, sehr stark kalkhaltiger, stark humushaltiger,

feinsandiger Schluff; schwach subpolyedrisches Gefüge

$\mathrm{G}_{\mathrm{o}} \mathrm{C}_{\mathrm{v}} \quad 15-40 \mathrm{~cm}$ graugelber, sehr stark kalkhaltiger, feinsandiger Schluff bis schluffiger

Analysen vgl. Tab. 5.

Feinsand; rostfleckig; ungegliedert.

Die hohen Kalk- und niedrigen Eisengehalte zeigen den geringen Verwitterungsgrad der Böden an. Die Gleymerkmale werden durch das über dem nahen Flinzuntergrund gestaute, zum Terrassenrand ziehende Grundwasser verursacht. Wo der Flinzuntergrund stärker ansteigt, finden sich Gleye mit Nässemerkmalen bis in die Krume.

Die hohen Humusgehalte in Profil 10 sind als Relikte eines vor der jüngsten Flußeintiefung durchlaufenen Anmoorstadiums aufzufassen.

g) Die Böden der Jüngeren Auenstufe (16)

Die Jüngere Auenstufe bildet das bis in die Gegenwart periodisch überflutete, von dichtem Auenwald bestandene Hochwasserbett des Lech. Die bis zur Errichtung der Wehre in den 40er Jahren ungebrochene Flußaktivität hat in diesem Bereich ein sehr unruhiges Relief hinterlassen. Flächen mit über $1 \mathrm{~m}$ mächtigen, vorwiegend schluffigen und feinsandigen Ablagerungen wechseln mit Stellen, an denen der Schotter bis an die Oberfläche tritt.

Die Böden der Jüngeren Auenstufe besitzen geringe bis sehr geringe Entwicklungstiefen und einen praktisch bis die Krume gleichbleibend hohen Karbonatgehalt. Die Humusbildung ist relativ gering. Eine Gefügeaggregation hat noch kaum stattgefunden. Profil 11 kennzeichnet etwa das Maximalstadium der Bodenbildung.
Profil 11:
R 16480
H 26820

Sandau N; flache Rinne, eben; Auwald (Erle, Hasel, Weide, Liguster)

J un g e r Ka lk a u e n b o d en (Kalkpaternia) aus Flußmergel;

Normal-Lechwasserspiegel ca. $2 \mathrm{~m}$ u. Fl.

$\mathrm{O}_{\mathrm{L}} \quad 1 \mathrm{~cm} \quad$ Laubstreu

$\mathrm{A}_{\mathrm{h}} \quad 0-10 \mathrm{~cm}$ grauer, sehr stark kalkhaltiger, humoser, feinsandiger Schluff; feinbröcke-

lig-krümeliges Gefüge (schwach ausgeprägt)

$\mathrm{AC}_{\mathrm{V}} \quad 10-25 \mathrm{~cm} \quad$ gelbgrauer, sehr stark kalkhaltiger, schwach humoser, feinsandiger Schluff

$\mathrm{G}_{\mathrm{O}} \mathrm{C}_{\mathrm{V}} \quad 25-80 \mathrm{~cm}+$ gelbgrauer, sehr stark kalkhaltiger, glimmeriger, feinsandiger Schluff und

Analysen vgl. Tab. 5. Sand im Wechsel über Kies; rostfleckig. 
Tabelle 5

A nalysen zu den Bodenprofilen $1-11$

\begin{tabular}{|c|c|c|c|c|c|c|c|c|c|c|c|c|c|}
\hline \multirow[b]{2}{*}{$\begin{array}{l}\text { Profil } \\
\text { Nr. }\end{array}$} & \multirow[b]{2}{*}{$\begin{array}{c}\text { Bodentyp } \\
\text { (Terrassenstufe) }\end{array}$} & \multirow[b]{2}{*}{$\begin{array}{c}\text { Hori- } \\
\text { zont }\end{array}$} & \multicolumn{3}{|c|}{ Korngrößen $\%$} & \multirow[b]{2}{*}{$\begin{array}{c}\mathrm{pH} \\
(\mathrm{KCl})\end{array}$} & \multirow{2}{*}{$\begin{array}{c}\text { Kar- } \\
\text { bo- } \\
\text { nate } \\
0 \%\end{array}$} & \multirow{2}{*}{$\begin{array}{c}\text { T- } \\
\text { Wert } \\
\text { (mval) }\end{array}$} & \multirow{2}{*}{$\begin{array}{l}\text { V- } \\
\text { Wert } \\
0 \%\end{array}$} & \multirow{2}{*}{$\begin{array}{l}\text { Org. } \\
\text { Subst. } \\
\\
0\end{array}$} & \multirow{2}{*}{$\begin{array}{l}\text { Verh. } \\
\mathrm{C} / \mathrm{N}\end{array}$} & \multirow{2}{*}{$\begin{array}{c}\mathrm{Fe}_{2} \mathrm{O}_{3} \\
\text { (dith.) } \\
0 \%\end{array}$} & \multirow{2}{*}{$\begin{array}{c}\mathrm{Fe}_{2} \mathrm{O}_{3} \\
(\mathrm{HCI}) \\
0 \%\end{array}$} \\
\hline & & & $\begin{array}{c}\text { Ton } \\
<0,002\end{array}$ & $\begin{array}{l}\text { Schluff } \\
0,002- \\
0,06\end{array}$ & \begin{tabular}{|c|} 
Sand \\
$0,06-$ \\
$2 \mathrm{~mm}$
\end{tabular} & & & & & & & & \\
\hline 1 & $\begin{array}{l}\text { Parabraunerde } \\
\text { (Stufe 6) }\end{array}$ & $\begin{array}{l}\mathrm{A}_{\mathrm{h}} \\
\mathrm{A}_{\mathrm{l}} \\
\mathrm{B}_{\mathrm{t}}\end{array}$ & $\begin{array}{l}25 \\
36\end{array}$ & $\begin{array}{l}31 \\
19\end{array}$ & $\begin{array}{l}44 \\
45\end{array}$ & $\begin{array}{l}4,0 \\
4,2 \\
6,2\end{array}$ & 1,4 & $\begin{array}{l}24,4 \\
17,3 \\
23,7\end{array}$ & $\begin{array}{l}15 \\
32 \\
76\end{array}$ & & & $\begin{array}{l}1,56 \\
1,76 \\
2,40\end{array}$ & $\begin{array}{l}2,20 \\
2,64\end{array}$ \\
\hline 2 & (Stufe 1) & $\mathrm{B}_{\mathrm{t}}$ & 35 & 32 & 33 & 6,6 & 7,8 & 20,2 & 89 & & & & \\
\hline 3 & $\begin{array}{l}\text { Ackerbraunerde } \\
\quad \text { (Stufe 3) }\end{array}$ & $\begin{array}{l}A_{p} \\
A B\end{array}$ & & & & $\begin{array}{l}6,4 \\
6,8\end{array}$ & $\begin{array}{l}\text { Sp. } \\
2,1\end{array}$ & $\begin{array}{l}14,5 \\
13,2\end{array}$ & $\begin{array}{l}81 \\
86\end{array}$ & 3,2 & 10,2 & $\begin{array}{l}1,48 \\
2,04\end{array}$ & \\
\hline 4 & $\begin{array}{l}\text { Braunerde } \\
\quad \text { (Stufe 8) }\end{array}$ & $\begin{array}{l}\mathrm{A}_{\mathrm{h}} \\
\mathrm{B}_{\mathrm{v} 1} \\
\mathrm{~B}_{\mathrm{v} 2} \\
\mathrm{BC}\end{array}$ & $\begin{array}{l}34 \\
37\end{array}$ & $\begin{array}{l}44 \\
38\end{array}$ & $\begin{array}{l}22 \\
25\end{array}$ & $\begin{array}{l}4,0 \\
4,2 \\
5,7 \\
7,1\end{array}$ & 30 & $\begin{array}{l}39,9 \\
32,2 \\
31,2\end{array}$ & $\begin{array}{l}32 \\
47 \\
72\end{array}$ & 6,9 & 13,5 & $\begin{array}{l}1,64 \\
1,72 \\
1,88 \\
1,56\end{array}$ & \\
\hline 5 & $\begin{array}{l}\text { Braunerde mit } \\
\text { Tondurch- } \\
\text { schlämmung } \\
\quad(\text { Stufe 10) }\end{array}$ & $\begin{array}{l}\mathrm{O}_{\mathrm{H}} \\
\mathrm{A}_{\mathrm{h}} \\
\mathrm{A}_{\mathrm{l}} \mathrm{B}_{\mathrm{v}} \\
\mathrm{B}_{\mathrm{tv}} \\
\text { IIBC }\end{array}$ & $\begin{array}{l}32 \\
39\end{array}$ & $\begin{array}{l}46 \\
41\end{array}$ & $\begin{array}{l}22 \\
20\end{array}$ & $\begin{array}{l}3,5 \\
3,5 \\
3,9 \\
5,8 \\
7,0\end{array}$ & 26 & $\begin{array}{l}31,1 \\
32,1 \\
34,8\end{array}$ & $\begin{array}{l}31 \\
59 \\
74\end{array}$ & 65 & 27,1 & $\begin{array}{l}1,48 \\
1,48 \\
1,84 \\
1,36\end{array}$ & $\begin{array}{l}3,84 \\
4,56\end{array}$ \\
\hline 6 & $\begin{array}{l}\text { Rendzina- } \\
\text { Braunerde } \\
\quad(\text { Stufe 10) }\end{array}$ & $\begin{array}{l}\mathrm{O}_{\mathrm{H}} \\
\mathrm{A}_{\mathrm{h}} \\
\mathrm{A}_{\mathrm{h}} \mathrm{B}_{\mathrm{V}} \\
\text { IIBC }\end{array}$ & $\begin{array}{l}30 \\
29\end{array}$ & $\begin{array}{l}49 \\
46\end{array}$ & $\begin{array}{l}21 \\
25\end{array}$ & $\begin{array}{l}3,0 \\
4,0 \\
5,3 \\
7,1\end{array}$ & 18 & $\begin{array}{l}34,3 \\
33,1\end{array}$ & $\begin{array}{l}42 \\
68\end{array}$ & $\begin{array}{l}55 \\
7,3 \\
5,9\end{array}$ & $\begin{array}{l}25,1 \\
12,8\end{array}$ & $\begin{array}{l}1,84 \\
1,64 \\
1,40\end{array}$ & \\
\hline 7 & $\begin{array}{l}\text { Mullrendzina } \\
\quad \text { (Stufe 12) }\end{array}$ & $\begin{array}{l}\mathrm{A}_{\mathrm{p}} \\
\mathrm{AC}\end{array}$ & & & & $\begin{array}{l}6,5 \\
6,9\end{array}$ & Sp. & $\begin{array}{l}25,9 \\
22,6\end{array}$ & $\begin{array}{l}86 \\
92\end{array}$ & . & & 1,24 & \\
\hline 8 & $\begin{array}{l}\text { Braunerde } \\
\quad \text { (Stufe 13) }\end{array}$ & $\begin{array}{l}\mathrm{O}_{\mathrm{F}, \mathrm{H}} \\
\mathrm{A}_{\mathrm{h}} \\
\mathrm{B}_{\mathrm{v} 1} \\
\mathrm{~B}_{\mathrm{v} 2} \\
\text { IIBC }\end{array}$ & $\begin{array}{l}33 \\
37\end{array}$ & $\begin{array}{l}38 \\
37\end{array}$ & $\begin{array}{l}29 \\
26\end{array}$ & $\begin{array}{l}3,8 \\
4,5 \\
5,1 \\
5,4 \\
7,1\end{array}$ & 30 & $\begin{array}{l}37,8 \\
30,6 \\
30,0\end{array}$ & $\begin{array}{l}33 \\
53 \\
71\end{array}$ & 62 & 24,8 & $\begin{array}{l}1,52 \\
1,60 \\
1,48 \\
1,08\end{array}$ & \\
\hline 10 & $\begin{array}{l}\text { Rendzinaartiger } \\
\text { Auenboden } \\
\text { (Stufe 15) }\end{array}$ & $\begin{array}{l}\mathrm{O}_{\mathrm{F}, \mathrm{H}} \\
\mathrm{A}_{\mathrm{h} 1} \\
\mathrm{~A}_{\mathrm{h} 2} \\
\mathrm{AC} \\
\mathrm{G}_{\mathrm{O}} \mathrm{C}_{\mathrm{v}}\end{array}$ & & & & $\begin{array}{l}6,8 \\
7,2\end{array}$ & $\begin{array}{l}48 \\
49 \\
55 \\
60\end{array}$ & 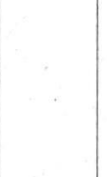 & & $\begin{array}{l}37 \\
16 \\
15\end{array}$ & 20,2 & $\begin{array}{l}0,56 \\
0,56 \\
0,64\end{array}$ & \\
\hline 11 & $\begin{array}{l}\text { Junger Kalk- } \\
\text { auenboden } \\
\text { (Stufe 16) }\end{array}$ & $A_{h}$ & & & & & 73 & & & & & 0,40 & 1,44 \\
\hline
\end{tabular}

M e th ode n : Korngrößenzusammensetzung nach KöHN; $\mathrm{pH}$-Wert: Messung mit Glaselektrode in $\mathrm{nKCl}$; Karbonate: gasvolumetrische Bestimmung mit 10\% iger $\mathrm{HCl}$; T-Wert nach VAGELERAlten/Kappen; V-Wert (Basensättigung) $=\mathrm{S} \cdot 100 / \mathrm{T}$; Organische Substanz nach Springer bzw. bei Karbonatgehalten über $10 \%$ nach Lichterfelde (Org. Subst. $=\mathrm{C} \cdot 1,724$ ); C/N-Verhältnis: Best. des Stickstoffs nach KJELDAHL; Dithionit-lösliches Eisen nach DEB; Gesamteisen im 1,15 HCIAuszug nach Reinhard-Zimmermann. 
Durch die Errichtung der Wehre wurde der Normalwasserspiegel des Lech örtlich bis zu $8 \mathrm{~m}$ gehoben, so daß ein Teil der Terrasse heute den typischen Wasserhaushalt der Gleye besitzt. Eine andere Vernässungsursache bildet der häufig hochanstehende, wasserstauende Flinzuntergrund, über dem das auf den Terrassen versickernde Niederschlagsoder Hangquellwasser zum Fluß abzieht. Profil 11 läßt einen solchen Grundwassereinfluß erkennen.

\section{Folgerungen aus den geomorphologisch-bodenkundlichen Untersuchungen im Hinblick auf die Tal- und Bodenentwicklung im Spät- und Postglazial}

Nach der vorausgehenden, zeitlich relativen Ordnung der Terrassen und der Ermittlung ihrer maximalen Bodenbildungen soll abschließend der Versuch unternommen werden, auf Grund der erzielten geomorphologisch-bodenkundlichen Ergebnisse zu einer Gliederung der fraglichen Zeitspanne zwischen Bildung der Niederterrassen und der mittelrömischen Terrasse, besonders aber zu einer Abtrennung des Spätglazials vom Postglazial zu gelangen.

\section{Die Grenze zwischen Spät- und Postglazial}

Die Böden der Stufe von Friedheim (8) hinken in ihrer Entwicklung erheblich hinter denen der Niederterrassen (1-6) nach. Betrachtet man die Mächtigkeit des Solums (Profile 1 und 4, S. 115 und 118) als Maßstab für den Verwitterungsgrad (BC-Horizonte zur Hälfte mitgerechnet), so verhält sich die Niederterrasse (6) zur Friedheimer Terrasse (8) wie $35: 22,5$. Auch die typologischen Entwicklungsunterschiede - hier Parabraunerde, dort Braunerde - kennzeichnen einen Entwicklungssprung.

Ein weiterer, wenn auch nicht mehr so markanter Entwicklungssprung liegt zwischen der Stufe von Friedheim (8) und der von Kaufering Bhf (9). Die Böden dieser beiden Terrassen sind zwar nur bedingt miteinander vergleichbar, da ihr Ausgangsmaterial in Korngröße und Karbonatgehalt differiert (hier Schotter mit ca. 75\% Karbonatanteil, dort Flußmergel mit 60-70\% Karbonatanteil), doch läßt sich der Entwicklungsunterschied etwa abschätzen, wenn man die Maximalbodenbildung der Friedheimer Terrasse (Profil 4) mit einer Bodenbildung der Kauferinger Terrasse mit sehr geringmächtiger Flußmergeldeckschicht (Profil 6) vergleicht.

Die Maximalbodenbildungen der anschließenden, jüngeren Flußmergelterrassen 9-14 unterscheiden sich hinsichtlich Entwicklungstiefe und typologischer Ausprägung von denen der Kauferinger Stufe und untereinander verhältnismäßig wenig. Der Entwicklungssprung zwischen Stufe 14 und 15 ist damit zu erklären, daß jene seit mindestens 2000 Jahren aus dem Grundwasserspiegel herausgehoben ist, während diese (mittelrömische Terrasse) bis in jüngste Zeit durch starke Lech-Hochwässer überschwemmt wurde.

Setzt man den Beginn des Gletscherrückzuges von seiner maximalen Eisrandlage mit etwa 20000 Jahren vor heute an (BüDel 1960), so stand für die Bodenbildung der Hauptniederterrasse eine Entwicklungszeitspanne von kaum weniger als 20000 Jahren zur Verfügung. Die Stufen 1-5 (Hauptniederterrasse bis Schongau-Peitinger Terrasse) fallen in die Zeit des Eisrückzuges von den äußeren Endmoränenwällen. Die nur wenig tiefer liegende Stufe 6 dürfte spätestens zur Zeit des Ammersee-Stadiums (nach H. Gross (1958) etwa 17000 Jahre vor heute) gebildet worden sein.

Der Entwicklungssprung der Bodenbildungen zwischen Stufe 6 und 8 läßt auf einen größeren Zeitabstand der Terrassenbildung schließen, in den eine Periode intensiver Verwitterung fällt. Als solcher ist nach dem Ammerseestadium die Alleröd-Wärmeschwankung bekannt. Die Bildung der Terrasse 8 würde somit in die Jüngere Tundrenzeit, d. h. in das ausgehende Spätglazial fallen.

$\mathrm{Zu}$ dem gleichen Ergebnis kommt man, wenn man von dem anderen Fixpunkt in der Terrassenabfolge, der mittelrömischen Auenstufe (15) ausgeht: Wurde die Stufe 15 zwi- 
schen dem ersten und vierten nachchristlichen Jahrhundert gebildet, so muß zwangsläufig ein Teil der Terrassen in der etwa 8 Jahrtausende umfassenden Zeitspanne zwischen Jüngerer Tundrenzeit und mittelrömischer Zeit gebildet worden sein. Die von Stufe zu Stufe nur um Nuancen verschiedenen Maximalbodenbildungen der Terrassen 9-14 sprechen für eine kontinuierliche Bodenentwicklungsperiode unter relativ ausgeglichenen klimatischen Bedingungen, wie sie nach dem Klimaumschwung vom Spät- zum Postglazial gegeben waren. Demnach ist die Grenze zwischen Spät- und Postglazial etwa zwischen Terrasse 8 und $9 \mathrm{zu}$ legen.

\section{Die Bodenentwicklung im Spät- und Postglazial}

Tabelle 6 zeigt die Bodenentwicklung seit Bildung der Hauptniederterrasse, wie sie sich nach den vorausgegangenen Darlegungen in den gegebenen Zeitrahmen einordnen läßt.

Tabelle 6

Die zeitliche Stellung der Lechterrossen und ihrer Bodenbildungen

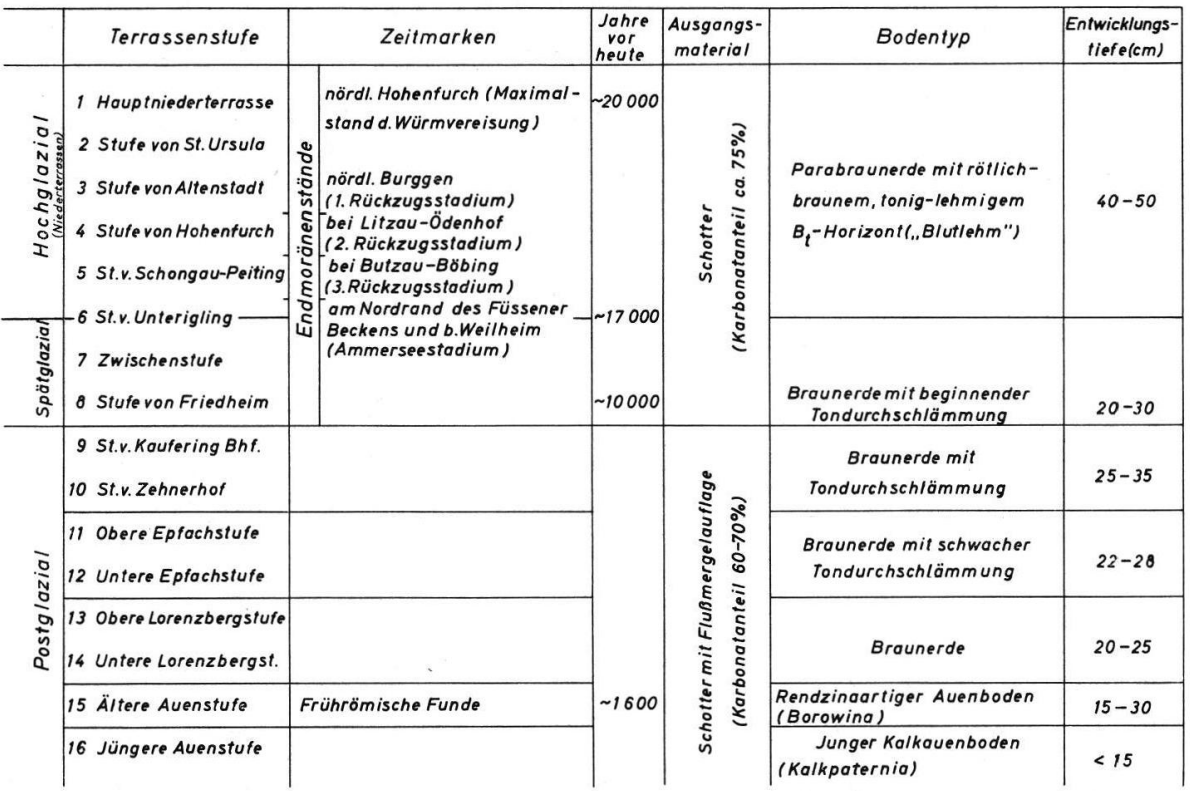

Am auffallendsten ist die ganz aus dem Rahmen fallende, starke Bodenbildung auf den hochglazialen Terrassen im Vergleich zu den jüngeren Terrassen.

Daß relativ tiefgründig verlehmte Böden nur auf den älteren Terrassenstufen der Alpenflüsse vorkommen, ist zumindest seit den Kartierungen von W. KoeHNE (1912, 1914, 1915, 1916) hinreichend bekannt. E. Kraus (1922) hielt die rötlichbraun gefärbten $\mathrm{B}_{\mathrm{t}}$-Horizonte für rotlehmähnliche Reste einer Bodenbildung während eines postglazialen Klimaoptimums und prägte für sie die Bezeichnung „Blutlehm“.

H. Harrassowitz (1926, S. 183) dagegen sieht in ihnen rezente Bodenbildungen, die auf den älteren Schotterterrassen auf Grund einer längeren Bildungszeitspanne lediglich mächtiger entwickelt sind.

G. Schmid (1962) hält die „Parabraunerden mit Blutlehmcharakter“ der würmglazialen Ablagerungen für interstadiale Bildungen, da nach seinen Angaben Parabraunerden auf jüngeren würmzeitlichen Moränen und Schotterfluren fehlen. Tatsächlich ist die Parabraunerde jedoch sowohl auf den jüngeren Niederterrassen als auch auf den unmittelbar 
am Alpenrand gelegenen Moränen die Maximalbodenbildung ('Th. DiEz 1964, 1966a, 1966b, 1967); allerdings geht die rötlichbraune Farbe des $B_{t}$-Horizontes, wie auch schon K. BRUNNACKER (1957) erwähnt, bei zunehmend feuchter werdendem Klima allmählich in ein rötlichliches Gelbbraun bis Braungelb über.

Von einer, den südbayerischen Verhältnissen entsprechenden Bodendifferenzierung zwischen spätglazialen und jüngeren Terrassen berichtet J. WeRnER (1964, S. 35-40) aus dem Bodenseegebiet. Seine Deutung - Hemmung der Bodenentwicklung auf den jüngeren Terrassen durch hohe Grundwasserstände bis ins Postglazial - ist für die unterschiedlichen Bodenbildungen auf den moränennahen Lechterrassen jedoch nicht brauchbar, $\mathrm{da}$ die bis in die Gegenwart anhaltende, tiefe Flußeinschneidung einen Grundwassereinfluß als Ursache für die unterschiedliche Bodenentwicklung ausschließt. Dagegen zeigt sich in der von J. WERNER erwähnten Gleichartigkeit der Bodenbildungen der älteren Niederterrassen der Argen eine interessante Parallelität zu den Bodenbildungen der Lech-Niederterrassen.

Von W. Moll (1965) werden die von ihm als „Rubefizierte Parabraunerden“ bezeichneten Böden auf Niederterrassen-Schotter des Oberrheintales im wesentlichen als Bildungen des postglazialen Klimaoptimums angesehen. In seiner Erklärung der Unterschiede in der Bodenentwicklung zwischen Niederterrassen und jüngeren Terrassen folgt er der Argumentation J. Werners bzw. K. Brunnackers.

K. Brunnacker (1957, S. 57-66) hat sich mit der Bildung der Parabraunerde aus Schottern der Alpenflüsse (von ihm "Schotterlehm" genannt) eingehend auseinandergesetzt. Er vertritt in der zitierten Arbeit die Ansicht, daß die Niederterrassen im Spätglazial durch Frostverwitterung und eine gewisse Entkalkung so stark aufbereitet wurden, daß die postglaziale Bodenbildung auf diesen Terrassen gegenüber jüngeren einen nicht mehr einzuholenden Entwicklungsvorsprung besaß. Die Bodenentwicklung sei im Spätglazial nicht über das Rendzina-Stadium hinausgelangt.

Gegen die Auffassung von K. Brunnacker sind auf Grund der in vorliegender Arbeit erzielten Ergebnisse folgende Einwände zu machen:

1. Wäre eine vorwiegend physikalische Verwitterung der Schotter im Spätglazial für die Bodenentwicklung erheblich, so müßte die Bodenbildung auf den von vornherein sehr stark aufbereiteten und weniger karbonathaltigen Flußmergeln der ältesten Postglazialterrassen zumindest ebenso stark sein als auf den hochglazialen Schotterterrassen.

2. Die etwa in die Jüngere Tundrenzeit zu stellende Friedheimer Terrassenstufe müßte, wenn die Hauptbodenbildung erst im Postglazial erfolgt wäre, eine den Niederterrassen viel näher stehende Bodenbildung zeigen, als den Terrassen des Postglazials. Das Gegenteil ist der Fall.

3. Die Parabraunerde auf den Niederterrassen und noch schöner die der petrographisch und altersmäßig vergleichbaren Jungmoränen zeigen neben den bereits erwähnten Verwitterungszapfen deutliche, auch den $\mathrm{B}_{\mathrm{t}}$ Horizont einbeziehende Taschenbildung und Verwürgungserscheinungen, die nur durch Kryoturbation oder Solifluktion zu erklären sind (Abb. 6).

Unterstellt man, daß letzte stärkere Frostbodenbildungen nur noch in der Jüngeren Tundrenzeit möglich waren, so ergibt sich daraus, daß die Parabraunerdebildung auf den hochwürmglazialen Terrassen und Moränen bereits in der Jüngeren Tundrenzeit im wesentlichen abgeschlossen sein mußte.

Die Annahme einer bereits im Spätglazial erfolgten Parabraunerdebildung ist nicht neu. K. Brunnacker (1959b) nimmt für die süddeutsche Parabraunerde aus Löß eine spät- bis frühpostglaziale Entstehung an. Neuerdings haben sich auch A. SEmmeL (1964) und W. Plass (1966) für eine präholozäne Entstehung der Bt-Horizonte in hessischen Mittelgebirgsböden ausgesprochen. 


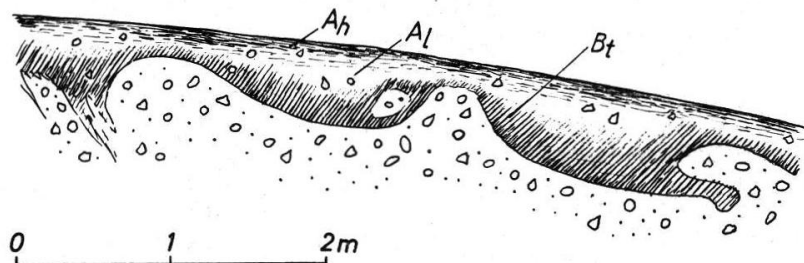

Abb. 6. Parabraunerde aus sandig-kiesiger Würm-Endmoräne mit starken Kryoturbationserscheinungen (Kiesgrube östlich Landsberg, R 23550, H 24380).

Eine starke Bodenbildung im Spätglazial, wie sie aus der Terrassengliederung und den pedologischen Befunden in dieser Arbeit gefolgert wird, wird aus klimatischen Gründen vielfach bezweifelt. Deshalb sollen im Folgenden kurz die spezifischen Bodenbildungsbedingungen des Untersuchungsgebietes erörtert werden.

Die Zeitspanne, welche die Niederterrasse den postglazialen Terrassen für die Bodenbildung voraus haben, kann mit mindestens 6000 Jahren veranschlagt werden. Die klimatischen Bedingungen während dieser Zeitspanne wechselten mehrfach zwischen kalttrocken und kühl-feucht (K. BRUnNacker 1959a). Für die Bodenbildung sind in erster Linie die feuchteren Klimaabschnitte von Bedeutung, die infolge der Höhenlage und unter dem Einfluß der Stauwirkung der Alpen im Untersuchungsgebiet schon immer dominiert haben dürften.

Die Bodenbildung aus karbonatreichen Gesteinen ist primär eine Frage der Lösungsverwitterung. $\mathrm{Da}$ die Karbonatlöslichkeit mit abnehmenden Temperaturen steigt - sie steigt nach C. Correns (1949) auf Grund der Löslichkeit und der Dissoziation der gasförmigen Kohlensäure bei einem Intervall von $0-20^{\circ} \mathrm{C}$ um das Doppelte - waren die niedrigen Temperaturen des Spätglazials der Lösungsverwitterung eher förderlich als abträglich. Allerdings darf die Vegetationsentwicklung nicht außer acht gelassen werden, da der Kohlensäuregehalt des Niederschlagswassers durch die $\mathrm{CO}_{2}$-reiche Luft des belebten Bodens stark erhöht wird (W. LAATsCH 1954, S. 44).

Nach F. Firbas (1949) ist bereits vor der subarktischen Bewaldung mit einer Pflanzenbedeckung zu rechnen, die den heutigen arktischen und alpinen Grasheiden und Matten geglichen haben dürfte. Noch vor der Allerödzeit sind Birken-Kiefernwälder im Gletschervorland nachgewiesen. „Der Verlauf der Pollenkurve, d. h. ihr allmählicher Anstieg, stimmt gut mit der schon von vornherein wahrscheinlichen Vorstellung überein, daß es im Laufe der Allerödzeit zu einer fortschreitenden Ausbreitung der Rohhumus- und zwergstrauchreichen Waldtypen gekommen ist" (FIRBAS 1949, S. 303). Gestützt auf die von H. Gross beobachtete spätglaziale Entkalkung von Ablagerungen der älteren Tundrenzeit, folgert F. Firbas, daß während der Allerödzeit offenbar eine chemische Verwitterung, Auswaschung und Entkalkung der Böden vor sich gegangen ist.

Gemäß den oben gemachten Ausführungen dürfte die Bodenbildung auf den älteren Lech-Niederterrassen bereits mit ihrem Trockenfallen gegen Ende des Hochglazials, wenn auch zunächst nur langsam, begonnen haben. Mit dem Aufkommen einer Vegetationsdecke steigerte sich die Entkalkungsgeschwindigkeit, um im Alleröd ihren Höhepunkt, einschließlich einer gewissen Tondurchschlämmung der bis dahin gebildeten, karbonatfreien Lösungsrückstände zu erfahren.

Wenn die Parabraunerde der Niederterrasse am Ende des Spätglazials bereits ausgebildet war, so erhebt sich die Frage, ob und wie sich in der nachfolgenden, insgesamt längeren Zeitspanne des Postglazials die Böden weiterentwickelt haben.

Eine gewisse Vertiefung des Solums im Postglazial ist mit Sicherheit anzunehmen, doch mußte sich der Entwicklungsprozeß in dem Maß verlangsamen, wie das Niederschlagswasser in den tonig-lehmigen Verwitterungshorizonten gespeichert und durch die 
Vegetation verbraucht wurde. Als Lösungsmittel und damit zur Verstärkung der Tiefenentwicklung trat das Niederschlagswasser nur noch partiell in Erscheinung. Das Stagnieren der Bodenentwicklung mit zunehmendem Alter führt zu einem Aufholen der Bodenbildungen jüngerer Oberflächen. Auf die scheinbare Gleichalterigkeit der Böden von Stufe 1-6 bezogen, bedeutet dies, daß die Bodenbildung der Stufe von Unterigling den Entwicklungsvorsprung der älteren Niederterrassenböden weitgehend aufholen konnte. Derselbe Vorgang wiederholte sich auf den postglazialen Terrassen und erklärt die geringen Bodenentwicklungsunterschiede der Stufen 9-14.

Die rötlichbraune Farbe der Parabraunerde aus Schotter hat nach unseren heutigen, vor allem pollenanalytisch fundierten Kenntnissen des spät- und postglazialen Klimas nichts mit einem warmzeitlichen Klima (vergleichbar etwa dem Mediterranklima der Terra rossa, wie E. Kraus annahm) zu tun. Dagegen scheinen die Faktoren W a s e r $\mathrm{h}$ a u shalt und $\mathrm{Z}$ e it auf die Bodenfarbe einen Einfluß zu haben. So zeigen die relativ trockenen Parabraunerden auf gut durchlässiger, sandig-kiesiger Jungmoräne ebenso wie auf den Niederterrassen, rötlichbraune Farben, während solche auf wasserstauender, schluffig-toniger Moräne gelbbraune Farben annehmen (Th. Diez 1967). Da die rötlichen Töne auf den jüngeren, hydrologisch den Niederterrassen gleichgestellten Terrassen nicht vorkommen, ein warmzeitliches Klima für das Spätglazial jedoch nicht angenommen werden kann, muß die Rotverfärbung auch eine Funktion des Alters der Böden sein.

Die Böden der postglazialen Terrassen - die bis in jüngste Zeit unter Grundwassereinfluß stehenden mögen hier außer Betracht bleiben - unterscheiden sich weniger durch die Entwicklungstiefe als durch den Grad der Verwitterung, Basenauswaschung und Tondurchschlämmung. Allen Maximalbodenbildungen ist trotz ihrer Flachgründigkeit (das karbonatische Ausgangsmaterial steht mindestens in einer Tiefe von $3 \mathrm{dm}$ an) eine sehr starke Versauerung und eine dementsprechende Basenverarmung der oberen Bodenhorizonte eigen. Rohhumusartige Humusformen können selbst bei Rendzina-Braunerden von weniger als $2 \mathrm{dm}$ Entwicklungstiefe auftreten. Die Gehalte an dithionitlöslichem Eisen spiegeln den abnehmenden Verwitterungsgrad der nichtkarbonatischen Komponente von den älteren zu den jüngeren Terrassen wider.

K. Brunnacker (1959a) gibt als terrestrische Bodentypen für die Postglazialterrassen an Lech und Isar nur Mullrendzinen ohne weitere Differenzierung an. Dies ist nur verständlich, wenn er sich — was bei der Waldarmut der Terrassenflächen erklärbar ist — auf Beobachtungen an landwirtschaftlich genutzten Böden stützt. Daß diese bei ihrer substratbedingten Flachgründigkeit die allein genetisch signifikanten, in Zentimeterbeträgen zu messende Horizontdifferenzierung nicht mehr zeigen können (und deshalb im morphologischen Sinn tatsächlich Mullrendzinen darstellen), wurde bereits ausgeführt (s. S. 115).

$\mathrm{Daß}$ die postglaziale Bodenbildung auf unterschiedlich alten Terrassen Entwicklungsunterschiede erkennen lassen muß, ist von vornherein anzunehmen. Erstaunlich ist allein die Tatsache, daß die Entwicklungsunterschiede nicht größer sind als in den Profilen 5-8 gezeigt wurde. Eine Erklärung für dieses Phänomen wurde bereits oben zu geben versucht.

\section{Schrifttum}

Brunnacker, K.: Die Geschichte der Böden im jüngeren Pleistozän in Bayern. Geologica Bavarica 34, München 1957. - - Zur Kenntnis des Spät- und Postglazials. Geologica Bavarica 43, München 1959 (1959a). - - Bemerkungen zur Parabraunerde. Geol. Jb. 76, 561-576, Hannover 1959 (1959b). - - Die geologisch-bodenkundlichen Verhältnisse in Epfach. Münchner Beiträge für Vor- und Frühgeschichte \%, München 1964.

BüDEL, J.: Die Gliederung der Würmkaltzeit. Würzburger Geogr. Arb. 8, Würzburg 1960.

Correns, C. W.: Einführung in die Mineralogie. Berlin, Göttingen, Heidelberg 1949. 
Diez, Th.: Die Böden. In ZACHER, W.: Erläuterungen zur Geologischen Karte von Bayern 1 : 25000 Blatt Nr. 8430 Füssen, München 1964. (123-138). - - Die Böden. In Stephan, W. und Hesse, R.: Erläuterungen zur Geologischen Karte von Bayern 1 : 25000 Blatt Nr. 8236 Tegernsee, München 1966 (1966a, 250-265). - - Die Böden. In Zacher, W.: Erläuterungen zur Geologischen Karte von Bayern 1:25 000 Blatt Nr. 8429 Pfronten, München 1966 (1966b, 182-193). - - Bodenkarte von Bayern 1 : 25000 Blatt Nr. 7931 Landsberg a. L. mit Erläuterungen. München 1967. - - Die Böden. In Ganss, O.: Erläuterungen zur Geologischen Karte von Bayern 1:25000 Blatt Nr. 8240 Marquartstein, München 1967.

Eimern van, J.: Klima. In Diez, Th.: Erläuterungen zur Bodenkarte von Bayern $1: 25000$ Blatt Nr. 7931 Landsberg a. L.-München 1967.

Firbas, F.: Waldgeschichte Mitteleuropas I. Jena 1949.

Graul, H.: Die Niederterrassenfelder im Umkreis von Basel. Eiszeitalt. u. Gegenw. 13, 181-196. Ohringen 1962.

Gross, H.: Die bisherigen Ergebnisse von C14-Messungen und paläontologischen Untersuchungen für die Gliederung und Chronologie des Jungpleistozäns in Mitteleuropa und Nachbargebieten. Eiszeitalt. u. Gegenw. 9, 155-187, Óhringen 1958.

Harrassowitz, H.: Studien über mittel- und südeuropäische Verwitterungsfragen. Klima-und Verwitterungsfragen 3. Teil. Geol. Rdsch. 17a (Steinmann-Festschrift), 122-210, Berlin 1926.

Knauer, J.: Geognostische Karte von Bayern 1 : 100000 , Blatt München West Nr. XXVII, Teilblatt Landsberg mit Erläuterungen. München 1929.

Koenne, W.: Blatt Pasing, Nr. 691 der Geol. Karte von Bayern $1: 25000$, München 1912. - Blatt Dachau, Nr. 667 der Geol. Karte von Bayern 1: 25 000, München 1912.

Koemne, W. \& Niklas, H.: Blatt Baierbrunn, Nr. 713 der Geol. Karte v. Bayern $1: 25000$, München 1914. - - Blatt Gauting, Nr. 712 der Geol. Karte v. Bayern 1:25 000, München 1915. - - Blatt Ampfing, Nr. 675 der Geol. Karte v. Bayern 1 : 25 000, München 1916.

KoHL, F.: Der nichtkarbonatische Anteil in südbayerischen Schottern und deren Böden. Geologica Bavarica 55, 360-371, München 1965.

Kraus, E.: Der Blutlehm auf der süddeutschen Niederterrasse als Rest des postglazialen Klimaoptimums. Geogn. Jh. 34, 149-221, München 1922.

L.Aatsch, W.: Dynamik der mitteleuropäischen Mineralböden. 3. Aufl. Dresden und Leipzig 1954.

Lieberoth, I.: Uber den Einfluß der Ackerkultur auf die Bodenentwicklung im sächsischen Lößgebiet. Albrecht-Thaer-Archiv 6, H. 1, 1962.

Moln, W.: Problematik rubefizierter und lessivierter Böden aus alpinem Material. Habilitationsschrift, Freiburg 1965.

Plass, W.: Braunerden und Parabraunerden in Nordhessen. Z. Pflanzenern. Düng. Bodenkunde 114, 1, 12-26, Weinheim 1966.

Rathjens, C.: Uber die Zweiteilung der Würmeiszeit im nördlichen Alpenvorlande. Peterm. Geogr. Mitt. Gotha 1951.

Richter, G.: Bodenerosion. Schäden und gefährdete Gebiete in der Bundesrepublik Deutschland. Forsch. z. deutschen Landeskde. 152, Bad Godesberg 1965.

Schaefer, I.: Die diluviale Erosion und Akkumulation. Forsch. z. deutschen Landeskde. 49, Landshut 1950. - - Geologische Karte von Augsburg und Umgebung 1:50 000, München 1956.

SchмID, G.: Über Parabraunerden mit Blutlehmcharakter aus diluvialen Schotter- und Moränenablagerungen Südbayerns. Z. Pflanzenern., Düng., Bodenkunde 99 (144), 1, 21-36, Weinheim 1962.

Seibert, P.: Die Pflanzengesellschaften im Naturschutzgebiet „Pupplinger Au“. Landschaftspflege und Vegetationskunde 1, München 1958.

Semmel, A.: Junge Deckschichten in den hessischen Mittelgebirgen. Notizbl. hess. Landesamt Bodenforsch. 92, 275-285, Wiesbaden 1964.

Troll, C.: Die jungglazialen Schotterfluren im Umkreis der deutschen Alpen. Forsch. dt. Landesu. Volkskunde 24, 4, 158-256, Stuttgart 1926. - - Die Rückzugsstadien der Würmeiszeit im nördlichen Vorland der Alpen. Mitt. Geogr. Ges. München 18, 281-292, München 1925. - - Über Alter und Bildung von Talmäandern. Erdkunde 4, 286-302, Bonn 1954.

Ulbert, G.: Der Lorenzberg bei Epfach. Die frührömische Militärstation. Münchener Beiträge zur Vor- und Frühgeschichte 9, München 1965.

WERnER, J.: Grundzüge einer regionalen Bodenkunde des südwestdeutschen Alpenvorlandes. Schriftenreihe der Landesforstverw. Baden-Württemberg 17, Freiburg 1964.

Manuskr. eingeg. 2. 2. 1968.

Anschrift des Verf.: Dr. Th. Diez, 8 München 22, Prinzregentenstraße 28, Bayerisches Geologisches Landesamt. 